TI 2015-071/II

Tinbergen Institute Discussion Paper
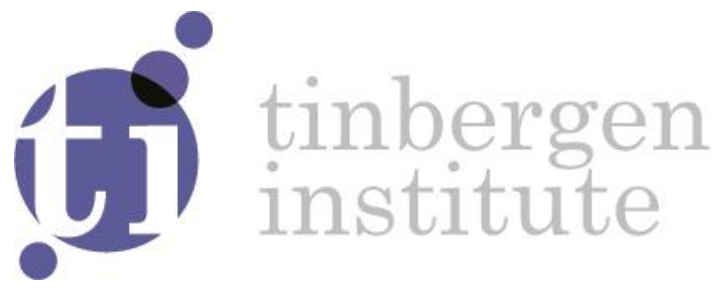

\title{
A Bargaining Experiment with Asymmetric Institutions and Preferences
}

\section{Revision: July 2018}

Aaron Kamm ${ }^{1}$

Harold Houba ${ }^{2}$

${ }^{1}$ New York University Abu Dhabi, United Arab Emirates

${ }^{2}$ Vrije Universiteit Amsterdam, Tinbergen Institute, The Netherlands 
Tinbergen Institute is the graduate school and research institute in economics of Erasmus University Rotterdam, the University of Amsterdam and VU University Amsterdam.

Contact: discussionpapers@tinbergen.nl

More TI discussion papers can be downloaded at http://www.tinbergen.nl

Tinbergen Institute has two locations:

Tinbergen Institute Amsterdam

Gustav Mahlerplein 117

1082 MS Amsterdam

The Netherlands

Tel.: +31(0)205984580

Tinbergen Institute Rotterdam

Burg. Oudlaan 50

3062 PA Rotterdam

The Netherlands

Tel.: +31(0)10408 8900 


\title{
A Bargaining Experiment with Asymmetric Institutions and Preferences
}

\author{
Aaron Kamm ${ }^{1}$ \& Harold Houba ${ }^{2}$ \\ ${ }^{1}$ New York University Abu Dhabi, United Arab Emirates \\ ${ }^{2}$ Vrije Universiteit Amsterdam and Tinbergen Institute, The Netherlands
}

July 2018

Third version. Previous version: TI 2015-071/II

Forthcoming in Social Choice and Welfare

\begin{abstract}
.
We report results from a laboratory experiment on strategic bargaining with indivisibilities studying the role of asymmetries, both in preferences and institutions. We find that subjects do not fully grasp the equilibrium effects asymmetries have on bargaining power and identify how subjects' observed behavior systematically deviates from theoretical predictions. The deviations are especially pronounced in case of asymmetric institutions which are modelled as probabilities of being the proposer. Additionally, in contrast to previous experimental work, we observe larger than predicted proposer power since subjects frequently propose and accept their second-preferred option. Quantal response equilibrium and risk aversion explain behavior whenever probabilities are symmetric, but less so when asymmetric. We propose the 'recognition is power' heuristic which equates bargaining power with recognition probabilities to explain these findings.
\end{abstract}

Please address correspondence to: Aaron Kamm, Division of Social Science, New York University Abu Dhabi, Building A5, PO Box 129188, Abu Dhabi, United Arab Emirates

Email: aaron.kamm@nyu.edu

\section{ACKNOWLEDGMENTS}

The authors are very grateful to the Associate Editor and two anonymous reviewers for valuable comments. We also thank Rebecca Morton, Matthew Embrey, Arthur Schram, Simon Siegenthaler, as well as (seminar) participants at the 2015 UECE Lisbon Meetings in Game Theory and Applications, the 2016 NYU Global Network Experimental Social Sciences Workshop, the University of Amsterdam and the Vrije Universiteit Amsterdam. Financial support from the University of Amsterdam Research Priority Area in Behavioral Economics and the Department of Econometrics and OR of the Vrije Universiteit Amsterdam is gratefully acknowledged. 


\section{Introduction}

Bargaining in committees to select among several candidates or alternatives is a widely occurring phenomenon. It is therefore of great interest and importance to understand which factors influence the decisions resulting from these bargaining processes. Consequently, a large empirical and theoretical literature has developed that studies this question. One of the seminal contributions to this strand of research is Baron and Ferejohn (1989) who propose a strategic bargaining framework to study these phenomena. In this framework in each round one player is randomly recognized to make a proposal and then the other players vote on it. If the proposal passes, the game ends and payoffs are realized, otherwise the game continues to the next round and again a proposer is chosen randomly.

Building on this work we study the effect of asymmetries - both in terms of institutions and in terms of preferences - on outcomes. It is crucial to understand the implications of asymmetries because these are probably the rule rather than the exception. In contrast to previous work we consider the effect of asymmetries in a setting with indivisibilities which are a common characteristic of bargaining within committees. For example, boards of companies appoint CEOs, parliaments choose Supreme Court judges and university committees hire new faculty. In many of these cases, the lack of side payments implies indivisibility. In such bargaining situations, parties involved are limited in trading off alternatives and instead have to decide between making a bold demand that claims their best alternative or a cautious demand that settles for their second preference. Given that both asymmetries and indivisibilities are very common it is crucial to understand the currently under-explored implications and interactions of indivisibilities and asymmetries on bargaining. In particular, institutions have a much larger effect on bargaining behavior in the presence of indivisibilities. Therefore, indivisibilities offer a fruitful setting for contributing to the relatively sparse literature on the effect of institutional asymmetries on bargaining behavior. Additionally, once we understand how asymmetric institutions affect bargaining processes we can think about what bargaining rules different players or a social planner would prefer. Therefore, valuable insights can also be gained for the literature on institution formation.

To investigate the effect of asymmetries we consider asymmetric preferences as well as asymmetric institutions. First, we study the case where players derive different monetary payoffs from their most favorable outcome and, second, we consider the situation where the institutional rules are asymmetric in the sense that some players are more likely than others 
to be recognized to make a proposal. The general question we consider in both cases is how these asymmetries influence the strategic interaction of players in the bargaining process. In case of asymmetric payoffs, the question is whether players can capitalize on the higher payoff from their preferred outcome or if competition between the players negates this seeming advantage. In case of asymmetric institutions the question is how the probabilities of being recognized translate into outcomes. For instance, we study whether a higher recognition probability implies higher expected earnings.

The natural first step in investigating the effect of asymmetries is to set up a model that reveals how they shape bargaining strategies and outcomes. In their seminal contribution Baron and Ferejohn (1989) study these phenomena in a perfectly divisible divide-the-dollar setting under majority voting. They consider asymmetries in institutions as asymmetries in agenda setting and propose to model these as asymmetric probabilities of being recognized as proposer. In their framework, there does not exist a Condorcet winner and parties are asymmetric in their recognition probabilities and in their role of either proposing or voting. Baron and Ferejohn show that the expected stationary equilibrium payoffs are insensitive to the distribution of recognition probabilities. Fréchette and al. (2005a) report empirical and theoretical results for an extension in which parties have asymmetric voting weights and recognition probabilities are either uniform or proportional to voting weights. Ceteris paribus of a representation in terms of unaffected minimal winning coalitions, they show that the expected equilibrium payoffs are insensitive to the distributions of recognition probabilities and voting weights. Fréchette and al. (2005a) also report empirical results from a laboratory experiment finding support for this insensitivity.

In general, the empirical and theoretical literature that studies bargaining situations under indivisibilities is relatively small. One plausible reason may be that such bargaining situations are much harder to analyze in the absence of a Condorcet winner. McKelvey (1991) and Herings and Houba (2016) consider a setting with three players, three indivisible alternatives, a Condorcet cycle under majority voting, ${ }^{1}$ and utilities that need not arise from divide-the-dollar settings. This is the simplest setting in which proposers face a hard choice between proposing their most or second-most preferred alternative, i.e. being bold or cautious. McKelvey (1991) characterizes the unique stationary equilibrium for the case of

\footnotetext{
${ }^{1}$ With three alternatives called $A, B$ and $C$, Condorcet cycles under majority voting feature either $A$ beats $\mathrm{B}, B$ beats $C$ and $C$ beats $A$, or the opposite sequence. Condorcet cycles rule out Condorcet winners and preserve the essence of no Condorcet winner in perfectly divisible divide-the-dollar bargaining.
} 
symmetric recognition probabilities allowing for asymmetric valuations while Herings and Houba (2016) extend this characterization to a setting where both recognition probabilities and valuations are allowed to be asymmetric. The analyses in these two papers reveal that, in contrast to the divide-the-dollar case, equilibrium play and expected payoffs are sensitive to changes in recognition probabilities and valuations. At the same time these effects can be quite subtle and equilibrium outcomes can react in ways that at first glance might seem unintuitive.

This raises the question whether the theoretical predictions are borne out by the data. There are good reasons why we might expect this not to be the case: players are often only boundedly rational and have non-selfish preferences. Then the subsequent question is how the observed behavior will differ from theoretical predictions. To answer these questions, we conduct a controlled laboratory experiment that allows us to test the theoretically predicted effects of asymmetries and to identify potential systematic deviations. ${ }^{2}$ We employ a setting with three players and three imperfectly divisible alternatives (as in McKelvey 1991, Herings and Houba 2016) since this shuts down fairness considerations to a large degree thereby making it easier to investigate whether subjects use their bargaining power as theoretically predicted and identify how they may deviate. In a two-by-two betweensubject design we vary recognition probabilities and payoffs. Each dimension of our design has a symmetric and an asymmetric version. In the treatments with symmetric recognition probabilities each player has a chance of $1 / 3$ to be the proposer while in the asymmetric treatments player 1 is the proposer with a probability of only $10 \%$ while players 2 and 3 each have a probability of $45 \%$ of being the proposer. These two sets of probabilities are the same as in Fréchette et al. (2005ab) who in a divide-the-dollar setting investigate the effect of recognition probabilities. An important difference to our experiment is that in their setting equilibrium payoffs are invariant to differences in recognition probabilities which makes it difficult to judge whether subjects understand the effect of recognition probabilities on bargaining power. In the treatment with asymmetric payoffs we substantially increase the attractiveness of player 2's most preferred alternative. In treatments with symmetric payoffs the monetary values of the most, second-most and least preferred alternatives for each player are permutations and therefore these treatments allow an interpretation as a divide-the-dollar setting. In contrast, in the asymmetric treatments where player 2 gets a substantially higher

\footnotetext{
${ }^{2}$ See McKelvey (1991), Fréchette et al. (2003), Fréchette et al. (2005a and b), and Diermeier and Morton (2006) for other experiments on strategic bargaining. Palfrey (2015) discusses experiments on bargaining in general.
} 
monetary payoff from her most preferred alternative than the other players get from their most preferred alternative such an interpretation is impossible since the game is no longer constant-sum. To our knowledge we are the first to report experimental evidence on bargaining behavior in such a setting.

From our four treatments two main hypotheses regarding the effect of asymmetries arise. First, payoff asymmetries have almost no effect on expected equilibrium payoffs. This implies that player 2 is unable to benefit from the higher payoff associated with her most preferred option. The reason is that the competition to be part of the winning coalition makes it impossible for her to sustain the advantage. Second, reducing player 1's recognition probability from $33 \%$ to $10 \%$ has no effect on her equilibrium payoffs, but substantially hurts player 3 even though her recognition probability increases from $33 \%$ to $45 \%$. The reason is that due to her lower recognition probability player 1 is easier to convince to become part of the winning coalition and therefore she is less often left out of the agreement while for player 3 the opposite is the case. The intriguing question is whether the subjects in the experiment are able to recognize this effect of asymmetries.

The analysis of the experimental data reveals two main findings: First, subjects show cautious bargaining by proposing and accepting their second-best alternative too often. Therefore, they underexploit their bargaining power because even though their best alternative has a higher than predicted probability of being implemented they nevertheless are more cautious than predicted. This finding might be caused by subjects' risk aversion and, paradoxically, implies proposer power that is larger than predicted. This is contrary to the common observation of lower proposer power, see for instance Palfrey (2015) and the references therein, and this difference is most likely caused by indivisibilities that force each proposer to choose between a bold demand for her best alternative or a cautious demand for her second-preferred option. Our empirical findings are in line with results in McKelvey (1991) who reports on an experiment with symmetric recognition probabilities testing the theoretical point predictions.

The second main finding that arises from the experiment is that for asymmetric recognition probabilities we observe systematic deviations from the model predictions. In particular, the player with the low recognition probability is much more cautious than predicted. In contrast, when recognition probabilities are symmetric, subjects' change in behavior when going from symmetric to asymmetric payoffs is more in line with the theory. The systematic deviations 
for asymmetric recognition probabilities do not only arise relative to the risk-neutral Nash equilibrium but also when a quantal response equilibrium -with risk-aversion and noise parameters estimated using experimental data- is used as a theoretical benchmark. Comparing observed behavior of the first and second half of the experiment did not show any evidence of learning. We therefore conclude that subjects have a harder time understanding the strategic implications of asymmetric recognition probabilities than of asymmetric payoffs. Humans are known to rely on heuristics, which would also explain the absence of learning. We propose the 'recognition is power' heuristic in order to explain behavior when probabilities are asymmetric: Subjects equate recognition probabilities with bargaining power. This would explain why the player with the lower recognition probability is much more cautious than predicted because she would think that her bargaining power is lower than it actually is.

The remainder of this paper is structured as follows: In the next section we present the experimental design; then section 3 describes the experimental results and compares these to Nash equilibrium predictions. Potential explanations for the observed deviations, in particular the quantal response equilibrium, are discussed in section 4 and section 5 presents the 'recognition is power' heuristic. Section 6 concludes with a summary of the results and a discussion of potential avenues for future research.

\section{Experimental design}

\section{$2.1 \quad$ The game}

Table 1: The payoff structure

\begin{tabular}{|c|c|c|c|}
\hline & Alternative I & Alternative II & Alternative III \\
\hline Payoff player 1 & 9 & 4 & 0 \\
\hline Payoff player 2 & 0 & $\beta$ & 4 \\
\hline Payoff player 3 & 4 & 0 & 9 \\
\hline
\end{tabular}

Notes. $\beta$ denotes player 2's payoff associated with her most preferred alternative, where $\beta$ is either 9 or 15 .

The game consists of a group of three players that has to decide which of three available options to implement. Bargaining proceeds as follows: In each round, all three players simultaneously submit a proposal (being one of the three alternatives in Table 1) they want the other players to vote on. After every player has submitted a proposal one of the proposals 
is randomly chosen to be voted on. ${ }^{3}$ Subsequently, the proposal of the selected player (the "proposer") is then communicated to the other two players which then can vote to accept or reject this proposal. The voting procedure is sequential: First the player who earns a higher payoff from the proposal gets to cast his vote. Given that we assume majority voting and that the proposer is implicitly assumed to support his own proposal, the proposal is accepted if the first voter accepts the proposal and acceptance ends the voting (and the bargaining). However, if the first voter rejects the proposal the second voter gets to cast his vote. If he votes yes a majority supports the proposal and it is accepted. Note that the last voter is only asked to vote if the first vote was a 'no', i.e. if she is pivotal. ${ }^{4}$ If a proposal is accepted the payoffs associated with the proposed alternative are implemented. If the proposal is rejected the game continues to the next period with probability $\delta \in(0,1)$ while with probability $1-$ $\delta$ bargaining breaks down and everyone receives a payoff of zero. In the experiment, we implement $\delta=0.9$, which corresponds to a risk of breakdown of $10 \%$ per round. The structure of payoffs (denoted in points) is shown in Table 1. Symmetric payoffs with a total of 13 points to be divided allow an interpretation as divide-the-dollar, while asymmetric payoffs lack such interpretation.

At this point we should note that while the structure of the bargaining process is similar to most previous experiments on strategic bargaining our payoff structure is different. While we employ a setting with a small set of possible proposals representing indivisibilities most of the literature considers a continuous divide-the-dollar setting. As we argue in the introduction, indivisibilities are a common but underexplored occurrence and our design aims at increasing our knowledge of behavior in such situations. Furthermore, given the goal of testing the predicted effect of asymmetries on behavior our design has two more advantages compared to a divide-the-dollar setting. First, in a setting with continuous alternatives recognition probabilities have no effect on payoffs (Frechette et al. 2005a) which makes it more challenging to test whether subjects understand the impact of asymmetric probabilities. Second, indivisibilities should reduce fairness considerations therefore making

\footnotetext{
3 This setup is strategically equivalent to selecting one player as the random proposer and also has several advantages. It keeps subjects engaged, all subjects have the same incentives in submitting their proposals as compared to a randomly selected proposer and more data on proposals are obtained.

${ }^{4}$ This implements the voting procedure in Herings and Houba (2016), who show that sequential voting eliminates the equilibrium in weakly-dominated strategies where both voters vote in favor of the proposal believing that the other will vote 'yes'. This reduction in equilibrium multiplicity has to be traded-off against a loss of data since we do not observe how the second voter would have voted when being non-pivotal. However, it is not clear how informative a non-pivotal vote is since such a vote has no impact on the outcome. Furthermore, the fact that subjects almost never accepted their least preferred option suggests that subjects understood the incentives they faced.
} 
it easier to test the theory. The reasoning is that given the binary nature of decisions it is quite expensive to be kind (i.e. to accept or propose one's middle option) while in a dividethe-dollar setting it is possible to be kind at relatively low costs to yourself.

\section{$2.2 \quad$ Treatments}

The experiment consists of four between-subject treatments that are constructed in the 2x2 configuration shown in Table 2. The first treatment dimension varies whether the alternatives are symmetric with respect to payoffs. In the symmetric case every player gets $9(4,0)$ points when her favorite (middle, worst) option is implemented, i.e. $\beta=9$. When payoffs are asymmetric player 2 gets 15 points instead of 9 points when her favorite alternative is implemented, i.e. $\beta=15$. The second treatment dimension varies the probability that a player will be the proposer in any given period. As in Fréchette et al. (2005a), we consider symmetric treatments in which each player has an equal probability to become proposer and asymmetric treatments in which player 1 is the proposer with a probability of $10 \%$ while players 2 and 3 each have a recognition probability of $45 \%$.

Table 2: Treatments

\begin{tabular}{|c|c|c|}
\hline & Symmetric payoffs & Asymmetric payoffs \\
\hline $\begin{array}{c}\text { Symmetric } \\
\text { recognition probabilities }\end{array}$ & SymPaySymRec & AsymPaySymRec \\
\hline $\begin{array}{c}\text { Asymmetric } \\
\text { recognition probabilities }\end{array}$ & SymPayAsymRec & AsymPayAsymRec \\
\hline
\end{tabular}

Notes. Cell entries give the treatment acronym used throughout this paper.

\subsection{Nash equilibrium}

Table 3 shows the resulting stationary subgame perfect Nash equilibria assuming riskneutrality (the equilibria are derived in Appendix A). We only report a player's probabilities of accepting and proposing her middle alternative since she will always accept her favorite alternative and neither propose nor accept her worst alternative. Therefore equilibria are completely described by the behavior regarding each player's middle option.

The equilibrium strategies have a general structure that is common in many bargaining models. In all treatments, a voter's continuation equilibrium payoff, which is the discounted value of this player's equilibrium payoff (i.e. $90 \%$ of the corresponding value in Table 3), determines the threshold for acceptance. A voter accepts her middle alternative if its payoff (of 4) is larger than this threshold while randomized acceptance requires equality. The lower 
the threshold, the weaker a responder is and the less bargaining power she has. For all players and in all equilibria, thresholds have a ceiling of 4, meaning no one is strong enough to reject their middle alternative with certainty. Furthermore, each player proposes the alternative with the highest expected continuation equilibrium payoff. This proposal is either the player's middle alternative, which will be immediately accepted by the voter whose best alternative it is, or her best alternative with possibly random acceptance and uncertain continuation of the bargaining. For all players and in all equilibria, the continuation equilibrium payoffs of proposing their best alternative have a floor of 4 , meaning everyone is strong enough to propose their best alternative with positive probability. If the proposer randomizes between her middle and best alternative, then she must face random acceptance of her best alternative, because otherwise it is strictly better to always propose her best alternative.

Table 3: Nash equilibrium

\begin{tabular}{|c|c|c|c|c|}
\hline & SymPaySymRec & SymPayAsymRec & AsymPaySymRec & AsymPayAsymRec \\
\hline \multirow{3}{*}{ Accept M } & $100 \%$ & $48 \%$ & $39 \%$ & $21 \%$ \\
& $100 \%$ & $23 \%$ & $58 \%$ & $18 \%$ \\
& $100 \%$ & $100 \%$ & $73 \%$ & $100 \%$ \\
\hline \multirow{2}{*}{ Propose } & $0 \%$ & $0 \%$ & $0 \%$ & $0 \%$ \\
M & $0 \%$ & $0 \%$ & $0 \%$ & $0 \%$ \\
& $0 \%$ & $13 \%$ & $0 \%$ & $10 \%$ \\
\hline \multirow{2}{*}{ Expected } & 4.3 & 4.4 & 4.4 & 4.4 \\
payoff & 4.3 & 4.4 & 4.4 & 4.4 \\
& 4.3 & 2.8 & 4.4 & 3.3 \\
\hline
\end{tabular}

Notes. Cell entries give the Nash equilibrium probability of accepting (proposing) the middle option and the expected equilibrium payoffs by treatment and player role (the first/second/third entry in each cell corresponds to player $1 / 2 / 3$ ) assuming risk-neutrality and continuation probability $\delta=0.9$.

As we can see from Table 3, in all but the completely symmetric treatment equilibria are in mixed strategies. In general, Nash equilibria with randomization have an unintuitive feature: The equilibrium strategies of all other players must make a randomizing player indifferent between all actions that are played with positive probability. Otherwise, shifting positive probability from non-optimal actions to better actions increases a randomizing player's expected utility. In textbook games, such as Matching Pennies, equilibria involving randomization resolve the endless merry-go-round argument why pure strategy equilibria do not exist. Below, we first explain the intuition of the pure equilibrium in the SymPaySymRec treatment. Then, we use treatment AsymPaySymRec to illustrate the merry-go-round 
arguments why specific pure strategies are not an equilibrium and provide intuition why randomization in Nash equilibrium is needed to resolve this endless argument. ${ }^{5}$

The SymPaySymRec treatment predicts that all players fully exploit their bargaining power by submitting their best alternatives and anticipating acceptance if their proposal is selected. The intuition is that voters prefer accepting middle alternatives with a payoff of 4 to the present value of obtaining one of the three alternatives with equal probability in the next round, which yields each voter an expected payoff of $0.9 \cdot \frac{13}{3}<4$. Each voter is therefore too weak to reject her middle alternative and concedes. This treatment shows that bargaining with indivisibilities and a Condorcet cycle may resolve disagreement efficiently without causing costly delay. Moreover, all players submit bold demands.

In AsymPaySymRec, the equilibrium in which all submit their best alternatives and all accept their middle alternatives breaks down because player 2 (given the higher payoff from her best alternative) now prefers to reject her middle option in the hope of getting her best option in the next period. Therefore, the next equilibrium candidate is a situation where everyone submits their best alternatives and only player 1 and 3 accept their middle alternatives. But this is not an equilibrium either, since in this situation players 2 and 3 will never agree and therefore player 1 will be part of any agreement. This gives her an incentive to reject her middle option and hope for getting her best outcome next period. Interestingly, this change in player 1's behavior induces player 2 to reconsider her strategy and return to accepting her middle option and therefore the next equilibrium candidate is a situation where everyone still submits their best alternatives and only players 2 and 3 accept their middle option. As it turns out by an argument parallel to the discussion of the previous case this situation is not an equilibrium either. And similarly, for the situation where it is player 3 rejecting her middle option is not an equilibrium. In equilibrium randomized acceptance resolves this merry-go-round argument; all players submit their best alternatives and all players randomly accept their middle alternative.

\section{$2.4 \quad$ Hypotheses}

From the equilibrium predictions in Table 3 and our two-by-two design, we derive two sets of hypotheses.

\footnotetext{
${ }^{5}$ The mechanism underlying the mixed strategies in SymPayAsymRec and AsymPayAsymRec is very similar and omitted.
} 
1. Effect of recognition probabilities

a. For players 1 and 2 asymmetries reduce the likelihood of accepting the middle option.

b. For player 3 asymmetries increase the frequency of accepting the middle option when payoffs are asymmetric.

c. Asymmetric recognition probabilities increase player 3's frequency of proposing the middle option and do not affect the other players' proposing behavior.

d. Asymmetric recognition probabilities reduce player 3's payoff and have no substantial effect on the other players' payoff.

2. Effect of payoff structure

a. For players 1 and 2 asymmetries reduce the likelihood of accepting the middle option.

b. For player 3 asymmetries decrease the frequency of accepting the middle option when probabilities are symmetric.

c. The payoff structure does not substantially affect proposing behavior.

d. Payoff asymmetries have almost no effect on expected equilibrium payoffs and the only substantial change is to player 3's payoff when the probabilities are asymmetric.

While some of the strategy changes might at first glance seem unintuitive (for instance, why does player 1 reject her middle option more frequently when she has a lower recognition probability), in general these all rely on the effect a parameter change has on the 'cost' of making a player accept a proposal. Recognizing these changes requires equilibrium reasoning that is rather complex. It is precisely this complexity that motivated us to investigate the robustness of the theoretical predictions in the laboratory and it raises the question how subjects' observed behavior might systematically deviate from theoretical predictions. These complexities notwithstanding we can provide a clear intuition for the changes in expected payoffs which then in turn imply the changes to acceptance and proposing behavior.

In terms of asymmetric recognition probabilities, it is very unintuitive that player 3 is hurt most, while the other two players are not substantially affected. There are two simultaneous effects at work causing this result. First, player 3's best alternative needs player 2's approval, while player 2's best alternative needs player 1's approval. Since the increase in her 
recognition probability makes player 2 stronger and more eager to reject player 3's best alternative (see Table 3), player 3 is now less likely to get her favorite alternative. The second effect is that player 1 , now being recognized with a lower probability, is the only one proposing to player 3 and therefore there is now a higher chance that player 3 will be excluded from the agreement. The combined effect implies that even though players 2 and 3 have the same recognition probability they will receive different equilibrium payoffs which highlights that recognition probabilities are not a sufficient statistic for bargaining power. The reason is that it does not only matter how often a player gets to propose but also to whom, and in this respect player 2 and 3 are asymmetric.

When considering asymmetries in payoffs we find that in case of symmetric recognition probabilities equilibrium payoffs do not differ substantially across treatments. The reason is the competition among the players to be included in the pair that eventually agrees. When recognition probabilities are asymmetric, payoff asymmetries do not balance each other and the effect of player 1's equilibrium strategy, who now accepts player 2's best alternative at a substantially lower rate, on player 3's payoffs is positive. The reason is that higher rates of disagreement between player 1 and 2, lead to an increased likelihood of future rounds in which player 3 may either propose or be proposed her middle alternative.

According to Table 3, the theoretical predictions that a player submits her middle alternative lie within the relative small interval of 0 to 13 percent, which may seem as weak quantitative predictions. Obviously, a design with parameters that generate larger differences in the theoretical predictions across treatments would be preferred. However, there is a stark tradeoff between generating variations in proposing versus accepting behavior because a positive probability of proposing one's middle option implies certain acceptance of one's middle option. The reasons is that if a player is willing to settle for her middle option in the advantageous position of being the proposer she will certainly settle for it when being proposed to. Therefore, we opted to aim for considerable variation in acceptance behavior which is easier to induce than a similar variation in proposing behavior. Furthermore, even the small interval of 10 to 13 percent implies some stark qualitative predictions. Player 1's reduction of her recognition probability will not lead induce this player to submit her middle option as a proposal. Similarly for player 2's increase of her recognition probability. It is only player 3 who should ever submit her middle option and only when recognition probabilities are asymmetric. Furthermore, we can also report that player 3's probability of submitting her middle option seems very sensitive to risk aversion. To be more precise, Table 
A.1 and A.2 of Appendix A illustrate that player 3's probability of proposing her middle option rapidly increases in the risk aversion parameter $\alpha \leq 1$ when the players have identical CRRA utility functions of the form $u(x)=x^{\alpha}$. Therefore, the reported 10 to 13 percent of Table 3 should be seen as theoretical lower bounds on these probabilities that are most likely to be higher in practice due to risk aversion.

\section{$2.5 \quad$ Experimental protocol}

The experiment was conducted at the CREED laboratory at the University of Amsterdam in December 2013 and February 2014 and implemented using php/mysql. ${ }^{6}$ Participants were recruited using CREED's subject database. In each of nine sessions, between 18 and 27 subjects participated. Most of the 225 subjects in the experiment were undergraduate students of various disciplines. ${ }^{7}$ Earnings in the experiment are in 'points', which are converted to euros at the end of the experiment at an exchange rate of 10 points $=1 €$. The experiment lasted on average 80 minutes and the average earnings were $19 €$ (including a $7 €$ show-up fee).

After all subjects have arrived at the laboratory, they are randomly assigned to one of the computers. Once everyone is seated they are shown the instructions for the first part of the experiment on their screen. ${ }^{8}$ After everyone has read these and the experimenter has privately answered all questions, a summary of the instructions is distributed. Then, all subjects have to answer quiz questions that test their understanding of the instructions. After everyone has successfully finished this quiz, the experiment starts. When everyone has finished part I the instructions for part II are shown on the screen and again a summary is distributed and a quiz has to be passed before part II begins. Finally, after everyone has finished part II the instructions for part III are shown on the screen and subjects make their decision for part III. At the end of the session, all subjects answer a short questionnaire and are privately paid their cumulative earnings from the three parts.

In order to induce subjects to think very carefully about their decision immediately for part I of the experiment the game was run as described above but with 10-times the payoffs. ${ }^{9}$ Subjects were informed that in this part they would participate in a bargaining game, that

\footnotetext{
${ }^{6}$ For screenshots of the interface as well as the text of the instructions and the summary handout, see Appendix F.

${ }^{7} 148$ of the 225 participants were students in business or economics.

${ }^{8}$ They are informed that there will be three parts in the experiment but not what these parts will entail.

${ }^{9}$ This does not have any effect on the equilibrium predictions, provided the risk-neutrality also holds at this payoff level.
} 
they stay the same player throughout the first part and that they will never meet the two other group members in part II and part III of the experiment. The game started in period 1 with subjects learning their role (player 1, 2, or 3) and applied the strategy method, i.e., everyone decided on their proposal before one proposal was randomly chosen to be voted on. If the first voter (the non-proposing group member that likes the proposal better) votes ‘yes’ part I of the experiment ends and the payoffs according to the implemented alternative are realized. If the first voter votes 'no' the second voter has to decide. If she accepts the first part ends and payoffs are realized. If she rejects, then with probability 0.9 the game moves to the next period, which proceeds exactly the same as period 1. With the remaining probability bargaining breaks down, part I ends and all group members earn zero points.

Table 4: Treatments

\begin{tabular}{|c|c|c|}
\hline & Symmetric payoffs & Asymmetric payoffs \\
\hline Symmetric & $\mathrm{N}=10$ & $\mathrm{~N}=5$ \\
recognition probabilities & 78 subjects & 45 subjects \\
\hline Asymmetric & $\mathrm{N}=7$ & $\mathrm{~N}=7$ \\
recognition probabilities & 51 subjects & 51 subjects \\
\hline
\end{tabular}

Notes. Cell entries give the number of independent matching groups $\mathrm{N}$ and subjects for each treatment.

Part II works in a way similar to part I, but the payoffs are not multiplied by ten and this part consists of 10 bargaining games. Each game works as described for part I but after each round groups are randomly re-matched and every subject is randomly assigned one of the three roles within the group. For econometric reasons this re-matching is not done using the complete group of subjects in the laboratory but is based on independent matching groups (i.e. subgroups) of size 6 or $9 .{ }^{10}$ Table 4 shows the number of independent matching groups and subjects for each of the four treatments. ${ }^{11}$

In part III we measure risk-aversion using the task proposed by Eckel and Grossmann (1998). Subjects have to choose one of seven lotteries with varying payoff for winning and losing but all with a winning probability of $50 \%$.

\footnotetext{
${ }^{10}$ Subjects were simply told that they would be rematched with other participants.

${ }^{11}$ The larger number of subjects in treatment SymPaySymRec is due to an oversight where one session that was supposed to be AsymPaySymRec was run as SymPaySymRec instead. This error was noticed immediately and was made up for by running a session of AsymPaySymRec the next day.
} 


\section{Results}

The theoretical predictions that a player always accepts her best option and that she neither proposed nor accepted her worst option are borne out by the data. ${ }^{12}$ Therefore, we focus the analysis of the results on a player's acceptance and proposing behavior with respect to her middle option. Our discussion focuses on an in-depth analysis of behavior observed in part II of the experiment and for an analysis of part I which leads to similar conclusions we refer to Appendix E. In a first step we present a within-treatment analysis that investigates whether observed behavior corresponds to the equilibrium predictions. As a second step we analyze differences across treatments and investigate the effect of asymmetries on subjects' behavior.

\subsection{Within-treatment analysis}

For treatment SymPaySymRec, where all players are completely symmetric, Figure 1 shows behavior that is quite close to the prediction of immediate agreement (i.e., players propose their best option and the other player for whom this is the middle option almost always accepts). Though all players sometimes reject the middle option, this only happens rarely and does not significantly vary by player (p-value: 0.29$).{ }^{13}$ Furthermore, sometimes a player proposes her middle option but this happens only occasionally and the frequency does not significantly vary across players (p-value: 0.61 ).

In treatment SymPayAsymRec, where player 1 has a lower recognition probability, we find systematic deviations from Nash equilibrium. As we can see, player 1 is proposing her middle option regularly while in the predicted equilibrium she should only propose her favorite option. For player 3 we observe the opposite, she proposes her middle option less often than predicted. This results in player 1 being significantly more likely to propose her middle option than player 3 (p-value $<0.01$ ) who in turn is significantly more likely to propose her middle option than player 2 (p-value: 0.04). With respect to the acceptance behavior in this treatment, we observe that behavior does not differ as much as predicted across players since players 1 and 2 accept their middle option more often than predicted.

\footnotetext{
12 Indeed, in line with theory players almost never proposed their worst option (14 out of 2697 decisions) and rarely accepted it (6 out of 173 decisions) and these frequencies do not vary much by treatment. Furthermore, a player almost never rejected her best option (1 out of 57 decisions).

${ }^{13}$ Unless mentioned otherwise all p-values are taken from a logit regression with proposing (accepting) the middle option as dependent variable and standard errors clustered at the matching group level. All regression results are reported in Appendix C.
} 
Furthermore, it is not the case that player 3 is most likely to accept her middle option. Instead player 1 has the highest acceptance rate ${ }^{14}$ while player 3 's behavior is statistically indistinguishable from player 2's behavior (p-value: 0.30).

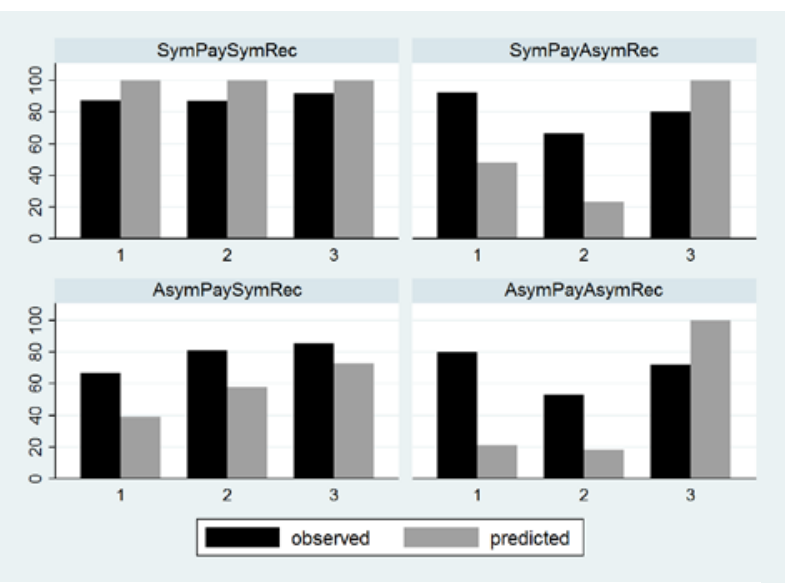

(a) fraction accepting middle option

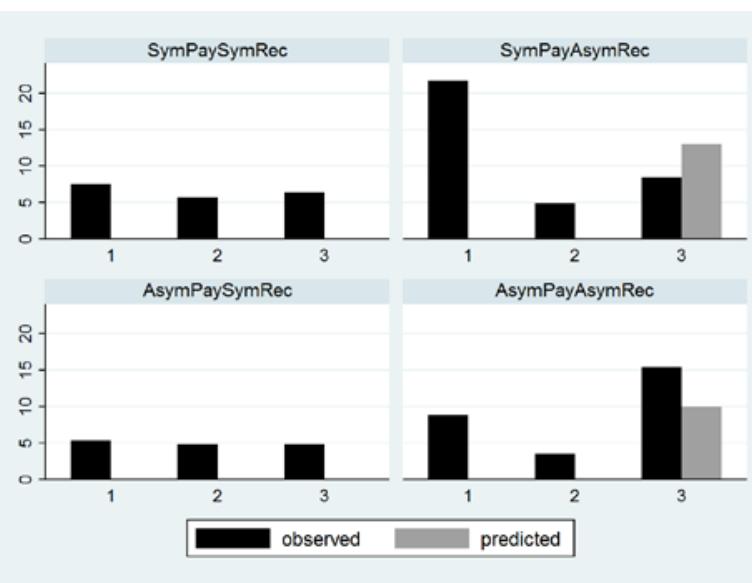

(b) fraction proposing middle option

Figure 1: Accepting and proposing one’s Middle Option in Part II

Notes. The figure shows the average fraction of accepting and proposing one's middle option observed in part II of the experiment split by role and treatment and compares them to the Nash equilibrium predictions.

For the treatment with asymmetric payoffs and symmetric recognition probabilities (AsymPaySymRec) we find that the proposing behavior is in line with the predictions since everyone is almost always proposing their best alternative and there is no difference across players (p-value: 0.88 ). For the accepting behavior we again find that the difference between players is smaller than predicted and that all players accept their middle options more often than predicted. We find that there is no significant difference between player 1 and 2 (pvalue: 0.09) or between player 2 and 3 (p-value: 0.41) but player 3 accepts her middle option significantly more often than player 1 does (p-value: 0.03). Overall, we find some support for the equilibrium predictions since proposing behavior and the ranking of acceptance rates is as predicted even though the differences in acceptance behavior are not as pronounced as predicted.

In the treatment were both payoffs and recognition probabilities are asymmetric (AsymPayAsymRec) we find that players 1 and 3 are proposing their middle option more frequently than predicted. This results in player 2 being significantly less likely to propose the middle option than player 1 (p-value: 0.04) who in turn has an insignificantly lower probability of proposing her middle option than player 3 (p-value: 0.09). For the acceptance

\footnotetext{
${ }^{14}$ The difference between players 1 and 2 is significant at the $1 \%$-level while the difference $1-3$ gives a p-value of 0.08 .
} 
behavior we find similar results to treatment SymPayAsymRec: players 1 and 2 accept their middle option substantially more often than predicted. Given that this effect is stronger for player 1 we observe a significantly higher acceptance rate by player 1 compared to player 2 (p-value: 0.02), which is not in line with the small predicted difference in acceptance rates. Furthermore, we do not find that player 3's acceptance rate is the highest but it is statistically indistinguishable from the other players' behavior (p-values are 0.49 and 0.15 for player 1 and 2, respectively). Overall, we find only limited support for the equilibrium predictions since it is not only player 3 who proposes her middle option and the pattern of acceptance rates is not in line with the theory for risk neutral players.

Combining all these results, two main observations arise. First, we find mixed support for the equilibrium predictions. On the one hand, the perfectly symmetric treatment corresponds nicely to the predictions and while asymmetric payoffs by themselves have less of an effect on behavior than predicted, the general pattern is still in line with the predictions. On the other hand, for the treatments with asymmetric recognition probabilities we find almost no support for the equilibrium predictions since the patterns of both acceptance and proposing behavior are far from what is predicted. Second, we find that subjects do not fully exploit their bargaining power when making their acceptance decision since they too often accept their middle option. This is in line with findings reported by McKelvey (1991) in a related experiment and implies a higher than expected proposer power. The subjects seem to overlook that other subjects accept their middle option too often and therefore forego exploiting this when proposing. This finding is in contrast to previous work on bargaining experiments which finds smaller than predicted proposer power, see e.g. Fréchette et al. (2003), Fréchette et al. (2005a and b), Diermeier and Morton (2006). We conjecture that the reason for these different findings is due to indivisibilities in our setting. These references consider perfect divisibility allowing for a smooth trade-off between kindness (i.e., giving up some of your own payoff in favor of others) and self-interest. In contrast, in our setting kindness is quite expensive, since it involves settling for your middle option. Therefore, with indivisibilities social preferences must be much stronger to have a noticeable impact on behavior. Given that social preferences are perceived to be the reason for the observation of lower than expected proposer power, this might explain our findings.

These observed deviations from the theory raise the question of how to explain them. In section 4 we discuss potential explanations and present an equilibrium analysis that relaxes the rationality assumption and also allows for risk-averse agents. As we will see this 
alternative model can account quite well for behavior when recognition probabilities are symmetric but is unable to capture all aspects of behavior when probabilities are asymmetric. Therefore, in section 5 we consider a heuristic where subjects equate recognition probabilities and bargaining power that we call the 'recognition is power' heuristic.

\subsection{Between-treatment analysis}

First, we consider the effects of going from symmetric to asymmetric recognition probabilities. As Figure 2 shows, for both payoff configurations this asymmetry is predicted to lead to a decrease in the acceptance rates of players 1 and 2 (Hypothesis 1a) while player 3's acceptance behavior should only be affected when payoffs are asymmetric (Hypothesis 1b).

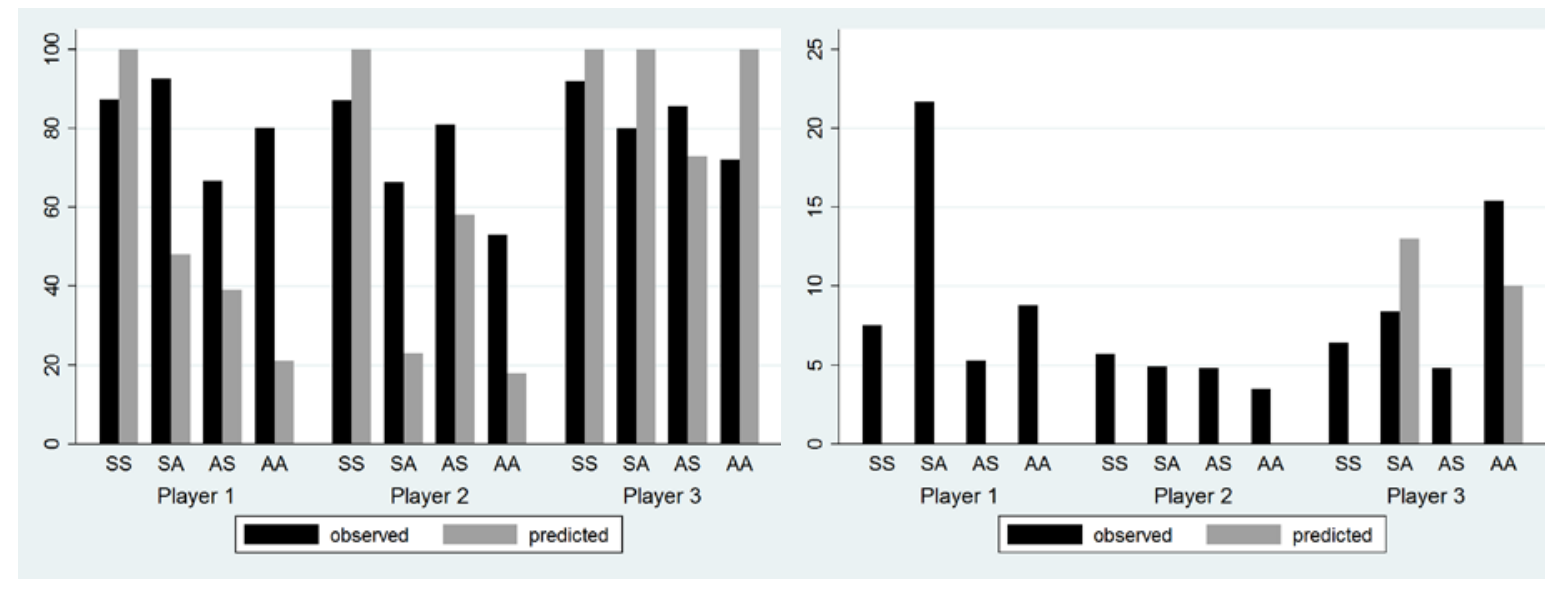

(a) fraction accepting middle option

(b) fraction proposing middle option

Figure 2: Accepting and proposing one's middle option in part II

Notes. The figure contrasts for each role and treatment the average observed fraction of accepting and proposing their middle option with the Nash equilibrium predictions. SS (SA, AS, AA) denotes the treatment with symmetric (symmetric, asymmetric, asymmetric) payoffs and symmetric (asymmetric, symmetric, asymmetric) recognition probabilities.

For player 2 we find support for Hypothesis 1a since she significantly reduces her probability of accepting the middle option (for symmetric payoffs, p-value $<0.01$; for asymmetric payoffs, p-value: 0.03) when recognition probabilities become asymmetric. For player 1 on the other hand, Hypothesis 1a is not supported since she (insignificantly) increases her acceptance rate instead of decreasing it (for symmetric payoffs p-value: 0.35 and for asymmetric payoffs 0.24 ). Additionally, Hypothesis $1 \mathrm{~b}$ finds only mixed support since independent of the payoff structure recognition probabilities do not significantly affect player 3's acceptance rate. While the insignificant effect (p-value: 0.10 ) was predicted for 
symmetric payoffs, for asymmetric payoffs we do not find the predicted increase in player 3’s acceptance rate but an insignificant decreases (p-value: 0.14).

The deviations from the theoretically predicted effect of asymmetric recognition probabilities are even more pronounced with respect to proposing behavior and overall our data do not provide much support for Hypothesis 1c. While, as predicted, the middle option is only regularly proposed when probabilities are asymmetric, the predicted increase in player 3's frequency of proposing her middle option is only significant when payoffs are asymmetric (p-values are 0.50 for symmetric and 0.00 for asymmetric payoffs). Furthermore, for symmetric payoffs we observe a significant increase in player 1's probability of proposing her middle option (p-value $<0.01$ ).

The observed deviations from theory in accepting and proposing the middle option also result in payoff consequences of asymmetric recognition probabilities that differ from the predicted effect. Hypothesis 1d states that asymmetric probabilities reduce player 3's average payoff while both player 1's and 2's payoff remain unchanged. This implies that having a lower recognition probability should not hurt player 1 . The data presented in Table 4 show that player 3 indeed suffers a significant reduction in payoffs but we also see that player 2 increases her payoff at the expense of player 1 (all changes are significant at the 1\%-level).

We now turn to the effects of going from symmetric to asymmetric payoffs. For accepting behavior we expect that asymmetric payoffs reduce player 1's and 2's propensity to accept their middle option (Hypothesis 2a) while only affecting player 3's behavior when recognition probabilities are symmetric (Hypothesis $2 b$ ). The observed behavior is broadly in line with these predictions but the decrease in the probability of accepting the middle option is only significant for player 1 (p-value: 0.05 for both symmetric and asymmetric probabilities) but not for player 2 (p-value 0.45 for symmetric and 0.20 for asymmetric). Furthermore, player 3 shows a lower probability of accepting the middle option when payoffs are asymmetric but this effect is not significant (p-value: 0.23 when probabilities are symmetric and 0.49 for the asymmetric case).

With respect to proposing behavior we expect payoff asymmetry to play no role (Hypothesis 2c). While this is what we observe when recognition probabilities are symmetric (p-values are $0.39,0.73$ and 0.42 for players 1,2 and 3 ) this prediction is not supported when recognition probabilities are asymmetric. Now payoff asymmetries significantly reduce 
player 1's probability of proposing her middle option (p-value <0.01) and significantly increases the frequency of player 3 proposing her middle option (p-value: 0.04). In sum, Hypothesis 2c is only supported for symmetric recognition probabilities.

For the average payoffs shown in Table 5 we expect to find no effect of asymmetric payoffs for players 1 and 2 and an increase for player 3 when the recognition probabilities are asymmetric (Hypothesis 2d). The predictions that player 2 is unable to exploit the increased payoff associated with her favorite option is not observed in the laboratory since player 2 can significantly increase her payoff (p-value $<0.01$ for both recognition probabilities). For asymmetric recognition probabilities, it is not player 3 that significantly increases her payoffs but player 1 (p-value: 0.02 for player 1 and 0.78 for player 3).

Table 4: Observed and predicted payoffs in part II

\begin{tabular}{|c|c|c|c|c|}
\hline & SymPaySymRec & SymPayAsymRec & AsymPaySymRec & AsymPayAsymRec \\
\hline \multirow{3}{*}{ observed } & 4.6 & 3.1 & 4.7 & 3.8 \\
& 4.0 & 6.3 & 5.3 & 8.4 \\
& 4.4 & 3.6 & 4.6 & 3.7 \\
\hline \multirow{3}{*}{ predicted } & 4.3 & 4.4 & 4.4 & 4.4 \\
& 4.3 & 4.4 & 4.4 & 4.4 \\
& 4.3 & 2.8 & 4.4 & 3.3 \\
\hline
\end{tabular}

Notes. Cell entries give the observed and predicted payoffs by treatment and player role (the first/second/third entry in each cell corresponds to player 1/2/3) assuming risk-neutrality and continuation probability $\delta=0.9$

Overall, from the between-treatment comparison a similar picture to the one found in the within-treatment analysis arises: Subjects do not react to asymmetries as predicted by theory and the deviations are more pronounced with asymmetric recognition probabilities than with asymmetric payoffs, indicating that subjects have more difficulties understanding the strategic effects of asymmetric recognition probabilities.

\section{Discussion}

The previous section begs the question as to the causes of these deviations from equilibrium. In the following we first discuss some well-known behavioral patterns and argue that they cannot explain the set of observed deviations. We then present an equilibrium analysis that considers two possible channels that might be at work -risk-aversion and noisy decisionmaking- and show that this analysis can explain some but not all the observed deviations. 


\subsection{Behavioral patterns}

It is by now well-know that a large number of behavioral patterns can explain deviations from the ideal of a selfish and rational homo oeconomicus. Chief among them are social preferences but as we have argued repeatedly throughout the paper we think that due to the indivisibilities they are only of limited importance for explaining our results. Furthermore, the 'taste for efficiency' (a preference for joint payoff maximization, Engelmann and Strobel 2004) as a special form of social preferences cannot explain our findings either. This preference would predict that when payoffs are asymmetric alternative 2 gets implemented more often but this is not the case. When recognition probabilities are symmetric, the percentage of the time that alternative 2 gets implemented drops from 31 to 27 when payoffs change from symmetric to asymmetric. Similarly when recognition probabilities are asymmetric, the percentage drops from 56 to 50 .

Another behavioral force that is frequently discussed in the context of bargaining is entitlement (see, for instance, Gächter and Riedl 2005) which in our setting would predict that proposers feel entitled to their preferred outcome since they are in a privileged position. Furthermore, if other players respect this entitlement they would be more likely to accept their middle option. For two reasons it is unlikely that entitlement effects can explain our findings. First, given the strategy method players have not yet won the right to be the proposer when submitting their choice which means that entitlement is at best anticipated which should reduce its salience. Second, entitlement would predict that players should very rarely propose their middle option which is not in line with the finding that, in particular, player 1 proposes her middle option more often than predicted in AsymRec.

While there are other behavioral mechanisms, like the inability of executing backward induction or competitive preferences, that are relevant when studying bargaining behavior we refrain from discussing all possible behavioral mechanisms and instead turn to another possible reasons for the observed deviations: bounded rationality.

\subsection{Quantal response analysis}

It is well established that humans may not be able to solve for the best-response as necessary for playing Nash equilibrium ${ }^{15}$ but are often observed to find a 'better-response', i.e. they

\footnotetext{
${ }^{15}$ In Appendix D, we investigate to what degree subjects' behavior can be explained by them best-responding to the other players' observed behavior in each independent matching group. The conclusions from this analysis are similar to the discussion in this section.
} 
tend to choose better options more often than worse options. This idea is captured by quantal response equilibrium (McKelvey and Palfrey 1995, 1998; Goeree et al. 2016), which assumes that the probability of choosing an action increases in the associated payoff. Previous experimental work (for instance, Goeree and Holt 2005) has shown that this equilibrium concept outperforms Nash equilibrium predictions in explaining experimental data. It also has been successfully applied to experiments on strategic bargaining (Battaglini and Palfrey 2014; Nunnari and Zapal 2016). ${ }^{16}$

In order to disentangle the role of bounded rationality and misspecified preferences we also allow for risk aversion when analyzing the quantal response equilibrium. Risk aversion might be relevant since the finding that players overall are more accommodating in their acceptance behavior than predicted would be in line with players being risk-averse since risk-averse players are less willing to take the gamble of rejecting their middle option in the hope of getting their favorite option in a future period. ${ }^{17}$

Table 6: Quantal response equilibrium

\begin{tabular}{|c|c|c|c|c|}
\hline & SymPaySymRec & SymPayAsymRec & AsymPaySymRec & AsymPayAsymRec \\
\hline \multirow{3}{*}{ Accept M } & $86 \%$ & $86 \%$ & $75 \%$ & $76 \%$ \\
& $86 \%$ & $69 \%$ & $75 \%$ & $59 \%$ \\
& $86 \%$ & $88 \%$ & $85 \%$ & $91 \%$ \\
Propose & $18 \%$ & $11 \%$ & $12 \%$ & $10 \%$ \\
M & $18 \%$ & $10 \%$ & $3 \%$ & $3 \%$ \\
& $18 \%$ & $24 \%$ & $18 \%$ & $36 \%$ \\
\hline \multirow{2}{*}{ Expected } & 4.3 & 3.7 & 4.7 & 4.5 \\
payoff & 4.3 & 5.2 & 6.2 & 7.4 \\
& 4.3 & 3.9 & 3.8 & 3.3 \\
\hline
\end{tabular}

Notes. Cell entries give the probability of accepting (proposing) one's middle option and the expected equilibrium payoffs by treatment and player role (the first/second/third entry in each cell corresponds to player $1 / 2 / 3$ ) for the quantal response equilibrium with $\alpha=.47$ and $\lambda=2.9$

We operationalize these two channels by using the experimental data to estimate, first, the parameter $\alpha$ of the CRRA utility function $x^{\alpha}$ and, second, the noise parameter $\lambda$ of the quantal response equilibrium. The results in estimates of $\alpha=0.47$ and $\lambda=2.9 .{ }^{18}$ The former is in line with previous work that estimated $\alpha$ 's in the range of 0.3 to 0.6 (see

\footnotetext{
${ }^{16}$ We also considered level-k (Nagel 1995, Stahl and Wilson 1995) as a way of modelling bounded rationality but level-k is unable to explain observed behavior.

${ }^{17}$ An analysis of decision-making at the individual level shows no systematic or substantial influence of riskaversion and gender on behavior. Detailed results of this analysis are presented in Appendix C.

${ }^{18}$ In Appendix B we present the underlying model specification.
} 
Battaglini and Palfrey 2014 and references therein). Table 6 shows the estimated quantal response equilibria.

Figure 3 shows the choice probabilities predicted by the quantal response equilibrium for the estimated parameters. Considering the acceptance decisions, we see that observed behavior is quite close to the quantal response predictions. Turning to the proposing decision, we see that when recognition probabilities are symmetric the fitted model does predict higher rates of proposing one's middle option than observed, which is opposite to the Nash equilibrium predictions. Furthermore, for asymmetric probabilities the fitted model is less accurate in capturing proposals. As when using Nash equilibrium as a solution concept, with symmetric payoffs player 1 is proposing her middle option more often than predicted. For player 3 we obtain a systematic deviation of doing so less often than predicted for both payoff structures, which differs from the deviations (one more often and one less often) from Nash equilibrium in Figure 1(b). Our estimated quantal response equilibrium indicates that subjects deviate from the rationality assumptions of Nash equilibrium and that errors in decision making are one important part of this deviation.

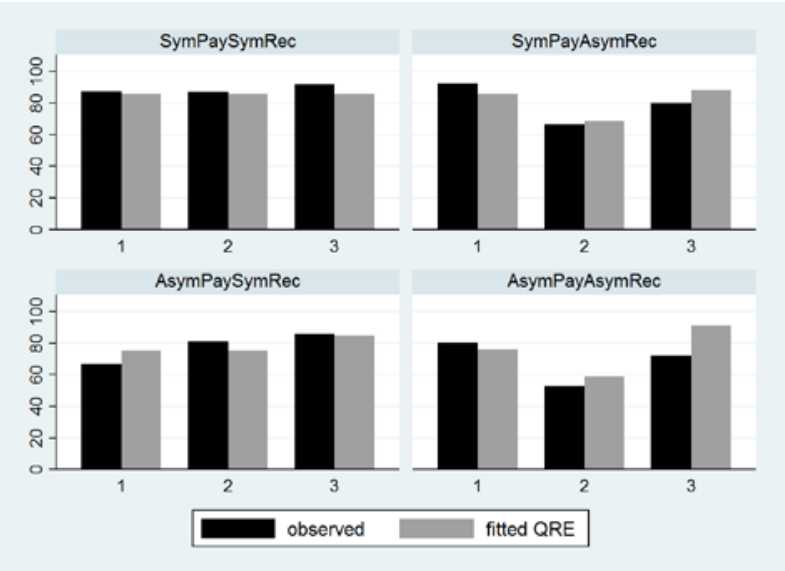

(a) fraction accepting middle option

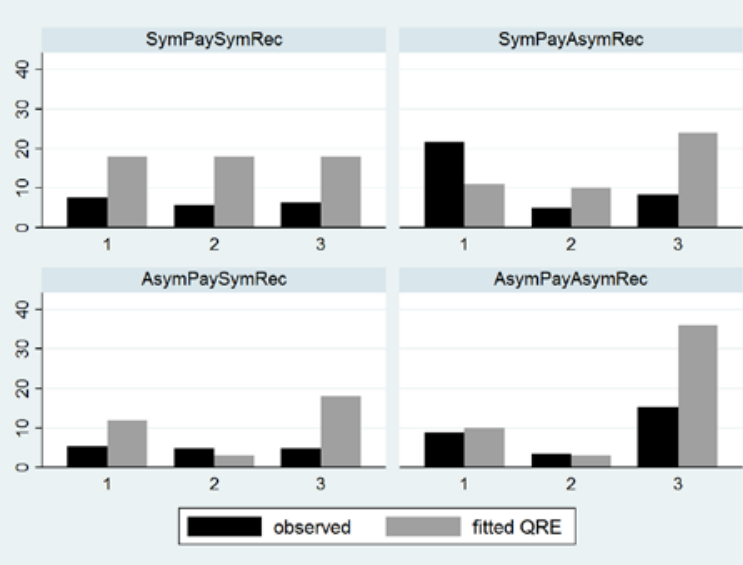

(b) fraction proposing middle option

Figure 3: QRE for accepting and proposing one’s middle option in part II

Notes. The figure shows the average fraction of accepting and proposing one's middle option observed in part II of the experiment split by role and treatment and compares them to the quantal response equilibrium for the estimated noise-parameter $\lambda=2.9$ and risk-aversion parameter $\alpha=.47$.

Table 7 compares the observed payoffs with the Nash equilibrium payoffs and the expected payoffs of the estimated quantal response equilibrium. Overall, the payoffs implied by QRE are closer to the observed payoffs than Nash equilibrium. In particular, QRE picks up that player 2 is the major beneficiary of the asymmetries, but when recognition probabilities are asymmetric the predicted effects are still substantially lower than observed. Furthermore, 
QRE picks up that player 1 is hurt by her lower recognition probability but the observed effect is substantially larger than predicted.

Table 7: Observed, Nash equilibrium and quantal response equilibrium payoffs in part II

\begin{tabular}{|c|c|c|c|c|}
\hline & SymPaySymRec & SymPayAsymRec & AsymPaySymRec & AsymPayAsymRec \\
\hline \multirow{3}{*}{ observed } & 4.6 & 3.1 & 4.7 & 3.8 \\
& 4.0 & 6.3 & 5.3 & 8.4 \\
& 4.4 & 3.6 & 4.6 & 3.7 \\
\hline \multirow{3}{*}{ Nash } & 4.3 & 4.4 & 4.4 & 4.4 \\
& 4.3 & 4.4 & 4.4 & 4.4 \\
\hline \multirow{3}{*}{ Quantal } & 4.3 & 2.8 & 4.4 & 3.3 \\
& 4.3 & 3.7 & 4.7 & 4.5 \\
& 4.3 & 5.2 & 6.2 & 7.4 \\
\hline
\end{tabular}

Notes. Cell entries give the observed and estimated quantal response equilibrium payoffs by treatment and player role (the first/second/third entry in each cell corresponds to player 1/2/3) assuming continuation probability $\delta=0.9$

Overall, we can conclude that while noisy decision-making and risk-averse subjects can explain most of the deviations from Nash equilibrium when probabilities are symmetric, the adjusted model still falls short in explaining all of the effects of asymmetric recognition probabilities.

\section{The 'recognition is power' heuristic}

The QRE analysis presented in the previous section suggests that the deviations from theory observed for asymmetric recognition probabilities are not only caused by risk aversion and mistakes in decision-making but that subjects exhibit a systematic bias when dealing with asymmetric recognition probabilities. One plausible source for this bias can be founded in the fact that humans often use heuristics in making decisions (Tversky and Kahneman 1974, Gigerenzer and Gaissmaier 2011) and therefore behavior might not be completely driven by expected payoffs. The use of a heuristic would also be consistent with the absence of learning throughout the experiment since a heuristic is a stable decision rule. We therefore propose the 'recognition is power' heuristic as a way to explain behavior with asymmetric recognition probabilities.

'Recognition is power' heuristic: Players equate recognition probabilities with bargaining power and therefore players with a high (low) recognition probability are bold (cautious) in their bargaining behavior. 
This heuristic can explain the deviations from both Nash equilibrium and QRE. Compared to the Nash equilibrium we find that player 1 is accepting and proposing her middle option too often which is consistent with her believing that due to the low recognition probability she has only little bargaining power. For QRE the main deviation observed for asymmetric recognition probabilities is that player 3 proposes her middle option too little which is consistent with her believing that due to the high recognition probability she is in a strong bargaining position which makes her more aggressive in her bargaining behavior.

\section{Conclusions}

Committees are often confronted with indivisibilities in bargaining. This is an important topic that is currently understudied. Our study is a first attempt to fill this void and suggests a promising and important direction for future research. We implement four treatments in a situation where three committee members bargain over three imperfectly divisible alternatives. To investigate the effect of asymmetries on bargaining behavior we vary the preferences (comparing symmetric payoffs to a situation where one player is advantaged) and institution (comparing symmetric recognition probabilities to a situation where one player has a lower probability of being recognized).

While subjects' behavior corresponds nicely to the equilibrium predictions when the game is perfectly symmetric, deviations from theory begin to appear when asymmetries are introduced. The two main deviations we observe are: First, subjects are more accommodating than expected and regularly accept their middle option; our analysis attributes this to risk-aversion. Second, subjects do not react to asymmetries in the way predicted by theory. While introducing asymmetric payoffs when recognition probabilities are symmetric leads to a change in the predicted direction (albeit less pronounced than expected), with asymmetric recognition probabilities substantial and systematic deviations from theory arise. The most pronounced aspect of these deviations is that the player with the low recognition probability is much more accommodating than predicted, since she accepts and proposes the middle option more often than theory prescribes. Furthermore, we did not find any evidence of learning. Our findings can partly be supported by a theoretical benchmark consisting of a quantal response equilibrium with risk-aversion and noise parameters estimated using our experimental data. Nevertheless, even this model with parameters estimated to fit observed behavior cannot fully explain the behavior in treatments with asymmetric recognition probabilities. A possible explanation for the results in 
treatments with asymmetric probabilities is that subjects use the 'recognition is power' heuristic which equates recognition probabilities and bargaining power. This would lead the 'weak' player with the low recognition probability to be more accommodating than predicted in Nash equilibrium and to one of the 'strong' players with a high recognition probability to be less accommodating than in the quantal response equilibrium that we fitted to the data.

As with all experiments one crucial question concerns the external validity of the results: what do our results imply beyond the specific set of asymmetries we have considered? While it is ultimately an empirical question what would happen for different parameter choices our results can offer some guidance. First, outcomes with asymmetric payoffs should be well explained by the theoretical model. This assessment is based on the observation that subjects in the experiment reacted to asymmetric payoffs as predicted. Second, in situations with asymmetric recognition probabilities players will rely on a heuristic equating nominal bargaining power (i.e. recognition probabilities) with real bargaining power and therefore (relative to theoretical predictions) outcomes will be biased against players with low recognition probabilities to the benefit of players with high recognition probabilities.

Another crucial dimension of external validity is whether the 'recognition is power' heuristic has applications beyond the bargaining game considered in this paper. We are aware of at least one important empirical regularity that could be explained with our heuristic: Gamson's Law (Gamson, 1961). Gamson’s Law describes the robust empirical finding that coalition governments tend to allocate government portfolios proportional to the seat shares parties are contributing to the government (for a survey of the empirical literature see Warwick and Druckman, 2006). The fact that nominal bargaining power instead of real bargaining power (i.e. pivotality when forming a winning coalition) matters for bargaining behavior is similar in flavor to the focus our subjects put on recognition probabilities. In particular, the order of who gets to attempt to form a government often proceeds in order of seat shares which implies a close relationship between proposer power and recognition probabilities.

Ultimately, the external validity of our results can only be tested by future work exploring bargaining with asymmetries. For instance, it would be very interesting to consider more complex bargaining settings, be it by increasing the number of players or by increasing the asymmetry across players. In such a stress test both the predictive power of the rational choice benchmark and our proposed heuristic could be further scrutinized. Another interesting design could rely on the theoretical work by Herings and Houba (2016) which 
demonstrates the absence of a straightforward mapping from the parameters of the game to equilibrium behavior. This makes it possible to set up treatments that share the same theoretical predictions but vary in their asymmetry which would be another way to test the effect of asymmetries. Finally, we think a very promising continuation of our study of asymmetric institutions would be to consider whether players are aware of the 'recognition is power' heuristic and try to exploit it when designing institutions. For instance, one could think about an experiment where in an initial stage players choose between different allocations of bargaining power. The question would then be whether players pick the theoretically optimal allocation, i.e. the one that gives them the largest real bargaining power, or whether they focus more on nominal bargaining power, i.e. recognition probabilities.

In conclusion, this paper offers a first step towards understanding the effect of asymmetric recognition probabilities and asymmetric institutions in general on bargaining behavior in the presence of indivisibilities. Given the importance and prevalence of strategic bargaining in determining political and economic outcomes we are looking forward to further work exploring the role of institutions and indivisibilities. Our results suggest that there is still much to learn. 


\section{References}

Baron, D. and Ferejohn, J. (1989) "Bargaining in Legislatures”, American Political Science Review, 83, 1181-1206.

Battaglini, M and Palfrey, T. (2014) “The Dynamics of Distributive Politics”, Economic Theory, 49, 739-777.

Diermeier, D. and Morton, R. (2006) "Experiments in Majoritarian Bargaining”, Social Choice and Strategic Decisions: Essays in Honor of Jeffrey S. Banks, Springer, Heidelberg.

Eckel, C., and Grossman, P. (2008) "Forecasting Risk Attitudes: An Experimental Study Using Actual and Forecast Gamble Choices”, Journal of Economic Behavior and Organization, 68, 1-17.

Engelmann, D. and Strobel, M. (2004) "Inequality Aversion, Efficiency, and Maximin Preferences in Simple Distribution Experiments”, American Economic Review, 94, 857-869.

Frechette, G., Kagel, J. and Lehrer, S. (2003) "Bargaining in Legislatures: An Experimental Investigation of Open versus Closed Amendment Rules”, American Political Science Review, 97, 221-232.

Fréchette, G., Kagel, J. and Morelli, M. (2005a) “Nominal Bargaining Power, Selection Protocol, and Discounting in Legislative Bargaining”, Journal of Public Economics, 89, 1497-1517.

Fréchette, G., Kagel, J. and Morelli, M. (2005b) “Gamson’s Law versus Noncooperative Bargaining Theory”, Games and Economic Behavior, 51, 365-390.

Gächter, S. and Riedl, A. (2005) "Moral Property Rights in Bargaining with Infeasible Claims”, Management Science, 51, 249-263.

Gamson, W. (1961) “A Theory of Coalition Formation”, American Sociological Review, 26, 373-382.

Gigerenzer, G. and Gaissmaier, W. (2011) “Heuristic Decision Making”, Annual Review of Psychology, 62, 451-482,

Goeree, J. and Holt, C. (2005) "An Explanation of Anomalous Behavior in Models of Political Participation”, American Political Science Review, 99, 201-13.

Goeree J., Holt, C. and Palfrey, T. (2016) Quantal Response Equilibrium: A Stochastic Theory of Games, Princeton University Press, Princeton.

Herings, P. and Houba, H. (2016) “The Condorcet Paradox Revisited”, Social Choice and Welfare, 47, 141-186. 
McKelvey, R. (1991) “An Experiment Test of a Stochastic Game Model of Committee Bargaining”, Contemporary Laboratory Research in Political Economy, University of Michigan Press, Ann Arbor.

McKelvey, R. and Palfrey, T. (1995) “Quantal Response Equilibria for Normal Form Games”, Games and Economic Behavior, 10, 6-38.

McKelvey, R. and Palfrey, T. (1998) “Quantal Response Equilibria for Extensive Form Games”, Experimental Economics, 1, 9-41.

Nagel, R. (1995) “Unraveling in Guessing Games: An Experimental Study”, American Economic Review, 85, 1313-1326.

Nunnari, S. and Zapal, J. (2016) “Gambler's Fallacy and Imperfect Best Response in Legislative Bargaining”, Games and Economic Behavior, 99, 275-294.

Palfrey, T. (2015) "Experiments in Political Economy" in The Handbook of Experimental Economics, Vol. II (Kagel and Roth, eds.), Princeton University Press, Princeton.

Stahl, D. and Wilson, P. (1995) "On Player's Models of Other Players: Theory and Experimental Evidence”, Games and Economic Behavior, 10, 218-254.

Tversky, A. and Kahneman, D. (1974) “Judgment under Uncertainty: Heuristics and Biases”, Science, 185, 1124-1131.

Warwick, P. and Druckman, J. (2006) "The Portfolio Allocation Paradox: An Investigation into the Nature of a Very Strong but Puzzling Relationship”, European Journal of Political Research, 45, 635-665. 


\title{
Online Appendices for
}

\section{A Bargaining Experiment with Asymmetric Institutions and Preferences}

\author{
Aaron Kamm ${ }^{1}$ \& Harold Houba ${ }^{2}$ \\ ${ }^{1}$ New York University Abu Dhabi, United Arab Emirates \\ ${ }^{2}$ Vrije Universiteit Amsterdam and Tinbergen Institute, The Netherlands
}

Appendix A: Nash equilibrium analysis 1

Appendix B: Quantal response analysis 8

$\begin{array}{ll}\text { Appendix C: Regression analysis for part II } & 10\end{array}$

Appendix D: Best responses against observed fractions 13

Appendix E: Behavior in part I 16

Appendix F: Instructions and screenshots of the experiment 17 


\section{Appendix A: Nash equilibrium analysis}

In this appendix, we apply the concept of stationary subgame perfect Nash equilibrium, abbreviated as Nash equilibrium, to a player's decision whether to propose her best or middle option and whether or not to accept her middle option. We do so, because it can be shown that each player's expected equilibrium payoff lies strictly between the utility of receiving her worst and best option. Therefore, if a player is proposed her best option, her best response is to accept it and if she is offered her worst option, she should reject it. Furthermore, proposing one's worst option is dominated by proposing the middle option, because both will be accepted.

Player E's strategy is then fully described by two probabilities; $2 \div$, the probability of accepting her middle option whenever it is proposed to her and $2, \hat{A}$, the probability of proposing her middle option and with complementary probability proposing the best option. We will use the following notation: Q denotes player Es utility from player Fs best option; $\ddagger$ is the probability that player Eis the proposer and < denotes the probability that the game continues to the next period when a proposal has been rejected.

With monetary payoff distributions in the experiment given by

$$
(9 ; 0 ; 4) ;(
$$

ZLWKHTXDOWRHLWKHURßESt option is EF 1 (F+1)'s middle (worst) option with the convention that $\mathrm{E}+1=4$ means 1 and $\mathrm{EF} 1=0$ means 3 .

The ex-ante expected utility $\ddot{\mathrm{E}}$ of player Eis then given by:

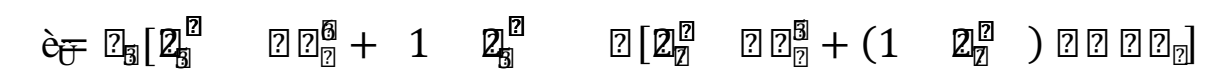

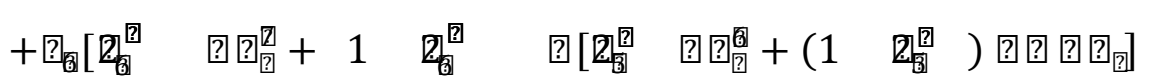

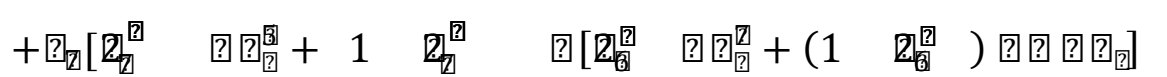

The ex-ante expected utility consists of the expected utility of possible and stochasticallyreached agreement in the current bargaining round plus the present value of the ex-ante expected equilibrium utility of continuing after the current bargaining round, i.e. $\ddot{E}_{<}$. The current-period expected utility depends on who is recognized as the proposing player and whether this player proposes her middle option that is accepted immediately, or her best option that is randomly accepted.

The equilibrium conditions for player i's probability of accepting her middle option are given by:

$$
\begin{aligned}
& \text {... } \\
& \text {... } ¥ 5 \text { ú } \ddot{E}^{\circ}{ }^{\circ} 6-\Pi € \text { 。 }
\end{aligned}
$$

The intuition is that if player Eaccepts (rejects) her middle option with positive probability, then the utility of her middle option cannot be smaller (larger) than the expected continuation utility of rejecting the offer. In particular, if player Erandomly accepts her middle option, 
$0<2 \div<1$, then both implications of (A.2) have to hold, and consequently, player Es exante expected equilibrium utility is given by $\ddot{\mathrm{E}}_{<}=<? 5 € \mathrm{Q}^{>5}$.

The equilibrium conditions for player Es probability of proposing her middle option are given by:

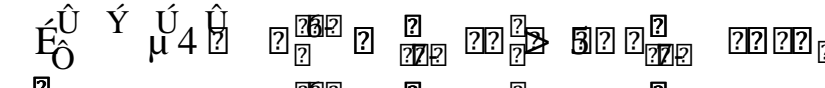

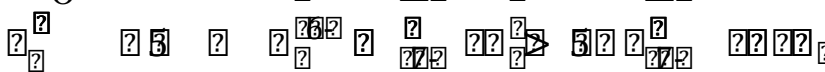

The intuition is that if player Erandomizes between proposes her middle (best) option with positive probability then the utility of her middle option, which will be accepted, cannot be lower (higher) than the expected utility arising from player Es best option being accepted with probability $2 \div 5$ by player EF 1 , whose middle option it is, and the complementary probability $1 \mathrm{~F} 2 \stackrel{\div}{2}$ that player Es best option is rejected and bargaining continues to the next round. In particular, if player EF 1 always accepts her middle option for sure, $2 \stackrel{\div}{<}=1$, then the implication of (A.3) cannot hold, and consequently, player Ealways proposes her best alternative for sure, $2, \hat{\mathrm{A}}=0$. Therefore, player Erandomly proposing her middle option requires sufficiently large probabilities of acceptance of player Es middle option by player EF 1.

Deriving Nash equilibria is a routine exercise that is tedious because it involves going through several cases, each related to the number of players with $2, \hat{A}$ has several subcases, each related to $2 \div>0$ or $2 \vdots<1$. We refer to Herings and Houba (2016) for an illustration of how to derive equilibria under $<=1$ and omit a detailed derivation in this appendix. For our numerical predictions, we will assume that the players have identical CRRA utility functions of the form $\mathrm{Q}(\mathrm{T})=\mathrm{T}, \ddot{\mathrm{Y}} \mathrm{Q} 1$. Table 3 in the main text shows the Nash equilibrium for the case $\ddot{Y}=1$ (risk neutrality) and $<=0.9$ as in the experiment. We briefly discuss each case.

\section{SymPaySymRec}

In the Nash equilibrium, each player always accepts her middle option, $2 \div=1$, and for each player it is then trivially optimal to always propose her best option, $2,{ }_{<}^{, \hat{A}}=0$. Consequently, there is immediate agreement and we have $\ddot{\mathrm{E}}_{<}=\frac{\ddot{\mathrm{E}}^{6-}>\ddot{\mathrm{E}}_{c}^{c}}{7}$. Under CRRA and $\ddot{\mathrm{Y}}=1, \ddot{\mathrm{E}}_{<}=\frac{57}{7}=$ 4.3 for all i's. More general under CRRA and $<=0.9$, the equilibrium condition for acceptance becomes $4 \mathrm{R}<€ \ddot{E}_{<}=<€ \frac{=>8}{7}$, which can be rewritten as $\ddot{\mathrm{Y}} € \ln \left(\frac{\overline{\bar{z}}}{8}\right) \mathrm{Q} \ln (3 €$ $<? 5$ F 1) and holds for $\ddot{Y}$ Q 1.047KLV UDQJHRI.IVLQFOXGHV WKHHQWLUHUDQJHRIULVNDY parameter values ( $\ddot{Y} \mathrm{Q} 1)$. 


\section{AsymPaySymRec}

7KH1DVKHTXLOLEULXPIRU.đVLQWKHUDQJHIURPWRLVDVIRQPRE \$andomly accept their middle option with a probability strictly between zero and one, and each player always proposes her best option, i.e. $2, \hat{,}, \hat{A}=0$.

By (A.2) random acceptance implies that $\ddot{E}_{<}=<? 5 € Q^{>5}$ and always proposing the best option requires $Q^{>5}$ Q $2 \underset{<}{\leftarrow} € Q+(1 F 2 \stackrel{\div}{\div}) €<€ \ddot{E}_{<}$. Under CRRA, this sets up the following set of equilibrium conditions

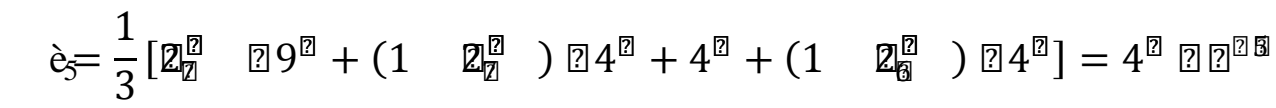

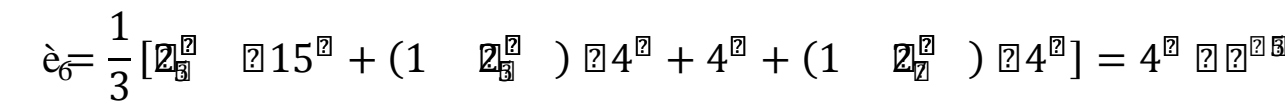

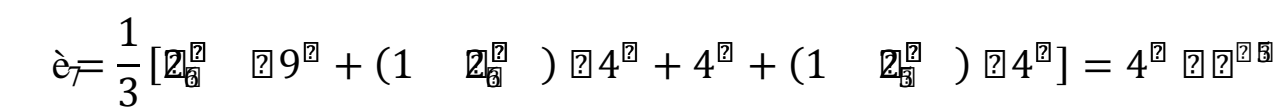

$$
\begin{aligned}
& 4 \text { Q } 2 \breve{\bar{j}} € 9+(1 \mathrm{~F} 2 \breve{\bar{j}}) €<€ \ddot{\mathrm{E}}_{5}
\end{aligned}
$$

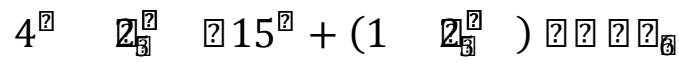

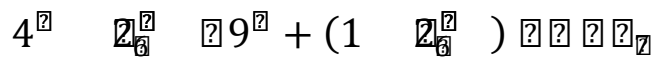

Because $\ddot{\mathrm{E}}_{<}=4 €<{ }^{? 5}$ and $2 \div-[0,1]$, each right hand side is at least 4 and these conditions hold independent of $\ddot{Y}$ and $<$. Under CRRA, $\ddot{Y}=1$ and $<=0.9$, this system solves

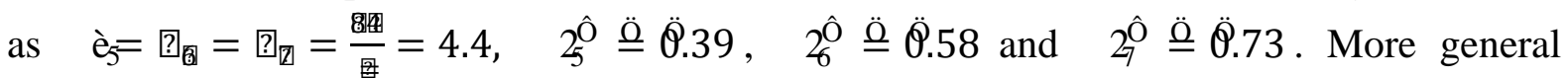
under CRRA and $<=0.9$, the equilibrium probabilities are given by

$$
\begin{aligned}
2 \ddot{\dot{5}} & =\frac{1}{3\left(\mathrm{~T}^{6} \mathrm{UF} 1\right)}\left(1+\mathrm{T}+\mathrm{T}^{6}\right) \\
2 \dot{\overline{6}} & =\frac{1}{3\left(\mathrm{~T}^{6} \mathrm{UF} 1\right)}(1+\mathrm{T}+\mathrm{TU}) \\
2 \ddot{\overline{\dot{*}}} & =\frac{1}{3\left(\mathrm{~T}^{6} \mathrm{UF} 1\right)}(1+\mathrm{U}+\mathrm{TU})
\end{aligned}
$$

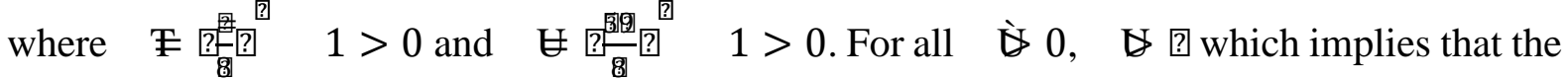

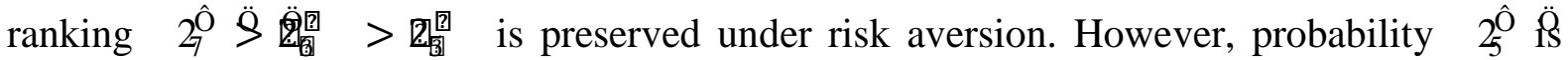
positive if and only if $\ddot{Y}>0.72$ (which ensures that $T^{6} U>1$ ), and probability $2 \dot{\bar{j}}$ is less than 1 if and only if $\ddot{Y}>0.92$. Therefore, it is player 3 who is critical in attaining the bound on the risk aversion parameter.

)RU.IVLQWKHUDQJHIUR $\$ 4$ to 0.92, the equilibrium changes slightly. All players still always propose their best option, player 1 and 2 randomize in accepting, and consequently $\ddot{\mathrm{E}}_{5}=\ddot{\mathrm{E}}_{6}=$ $4 €_{<} ? 5$ as before, and $2 \breve{\bar{j}}=1$, which by (A.2) imposes the equilibrium condition $\ddot{E}_{7} \mathrm{Q}$ $4 €<? 5$. This gives the following set of equilibrium conditions 


$$
\begin{aligned}
& \ddot{\mathrm{E}}_{5}=\frac{1}{3}[9+4+(1 \mathrm{~F} 2 \stackrel{\check{6}}{\overline{6}}) € 4]=4 €<? 5
\end{aligned}
$$

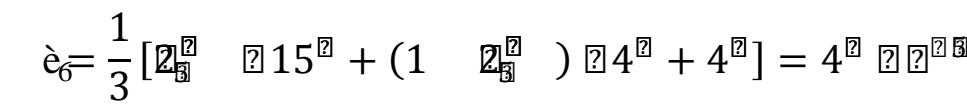

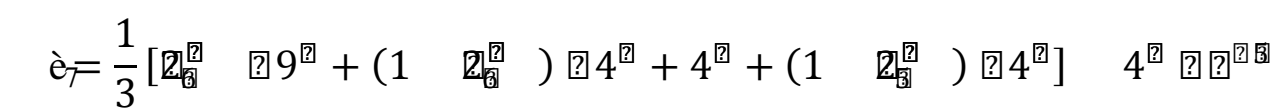

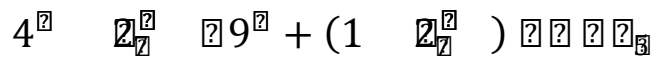

$$
\begin{aligned}
& 4 \text { Q } 2 \frac{\vdots}{5} € 15+(1 F 2 \stackrel{\doteqdot}{5}) €<€ \ddot{E}_{6}
\end{aligned}
$$

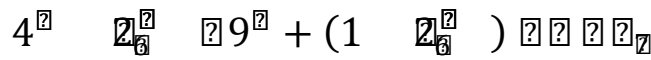

By previous arguments, the $4^{\text {th }}$ and $5^{\text {th }}$ conditions automatically hold. From the first two equations we obtain

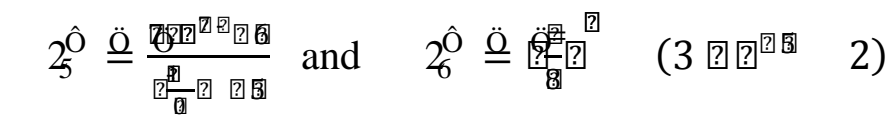

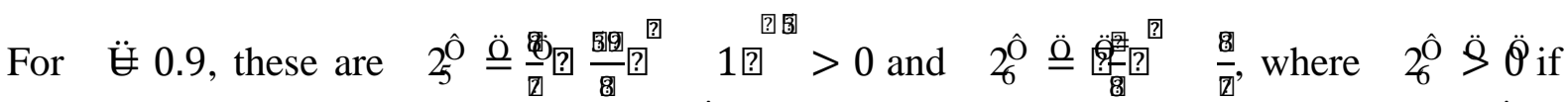
and only if $\ddot{Y}>0.35$. The probability $2 \stackrel{\dot{5}}{ }$ is less than 1 if and only if $\ddot{Y}>0.64$, and $2 \dot{\overline{6}}$ is less than 1 for all $\ddot{Y} Q$ 1.00. Player 3's equilibrium condition for accepting holds for $\ddot{Y}<0.92$ and this player's bound on his equilibrium payoff holds if and only if $\ddot{Y}>0.63$. To summarize, for $\ddot{Y}-[0.64,0.92]$ this is an equilibrium and it is now player 1 who is critical in attaining the bounds on the risk aversion parameter.

Note that the expected payoffs $\ddot{E}_{5}$ and $\ddot{E}_{6}$ are still equal, indicating equal bargaining power, while $\ddot{E}_{7} \mathrm{Q} 4 €_{<}{ }^{? 5}$ indicates that risk aversion makes player 3 loose bargaining power

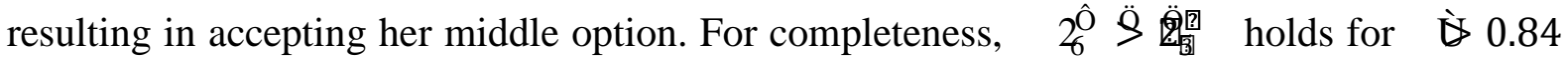
and for lower values the order reverses.

To summarize, the Nash equilibrium reported in Table 3 is robust for $0.92<\ddot{Y}$ Q 1 , its ranking

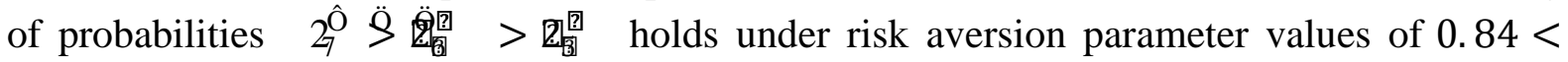
$\ddot{\mathrm{Y}} \mathrm{Q} 1$, and it is player 3 who is critical in attaining the bounds on the risk aversion parameter.

\section{SymPayAsymRec and AsymPayAsymRec}

In these two treatments, player 3 proposes her middle option with positive probability, but accepts her middle option for sure. In the equilibrium, player 1 and 2 always propose their best option and randomly accept their middle option. Formally, $2_{5}^{\hat{\mathrm{A}}}=2_{6}^{, \hat{\mathrm{A}}}=0,0<2 \frac{\dot{\overline{5}}}{\text {, }}$

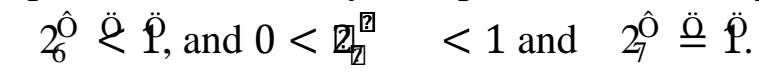

Like before, random acceptance imposes $\ddot{\mathrm{E}}_{5}=\ddot{\mathrm{E}}_{6}=4 € €_{<} ? 5$ and player 3's certain acceptance of her middle option requires $\ddot{E}_{7} \mathrm{Q} 4 €_{<}{ }^{? 5}$. Through (A.3), randomly proposing by player 3 imposes $2 \stackrel{\breve{6}}{€ 9}+(1 \mathrm{~F} 2 \stackrel{\breve{6}}{6}) €<€ \ddot{E}_{7}=4$ and always proposing their best

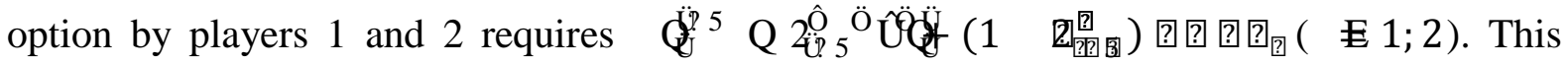
leads to the following set of equilibrium conditions 


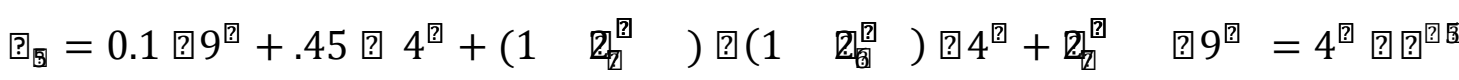

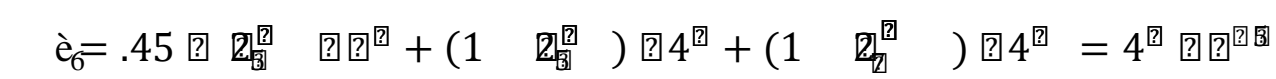

$$
\begin{aligned}
& \ddot{\mathrm{E}}_{7}=.55 € 4+.45 €\left(1 \mathrm{~F} 2 \frac{\leftarrow}{\overline{5}}\right) €<€ \ddot{\mathrm{E}}_{7} \mathrm{Q} 4 €<? 5 \\
& 4 \mathrm{Q} 1 € 9+0 €<€ \ddot{\mathrm{E}}_{5}
\end{aligned}
$$

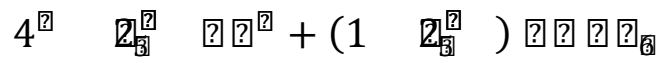

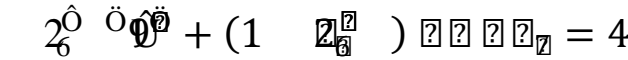

By previous arguments, the $4^{\text {th }}$ and $5^{\text {th }}$ conditions automatically hold. Solving the nonlinear system of equalities is to solve for three probabilities and player 3 's equilibrium payoff simultaneously. Solving is tedious and we just mention some of the intermediate steps. We implemented the change of variables $1 \mathrm{~F} 2 \div \quad /, \hat{\mathrm{A}} \quad \mathrm{E}=1,2,3$, and obtained

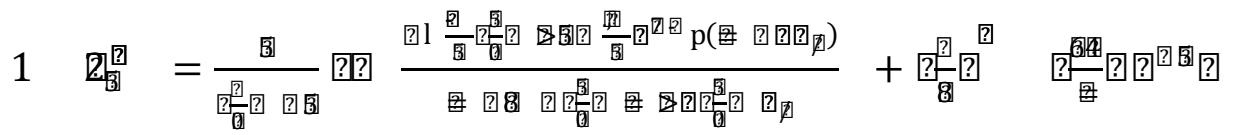

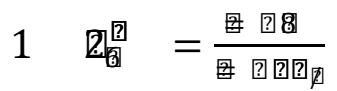

$$
\begin{aligned}
& 1 \mathrm{~F} 27^{\hat{\mathrm{A}}}=\frac{64}{=}<{ }^{5} \mathrm{~F} \underset{8}{@} \mathrm{~A}+(1 \mathrm{~F} 2 \stackrel{\vdots}{\overline{5}}) @ \frac{\mathrm{A}}{\mathrm{F}} 1 \mathrm{~A}
\end{aligned}
$$

Equilibrium payoff $\ddot{\mathrm{E}}_{7}$ is a root of $\mathrm{TE}_{7}^{6}+\mathrm{UE}_{7}+\mathrm{V}=0$, where

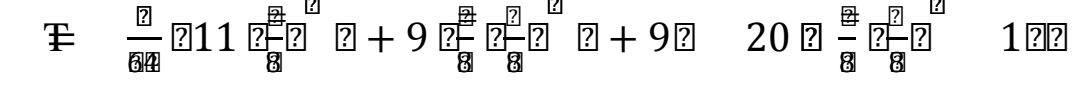

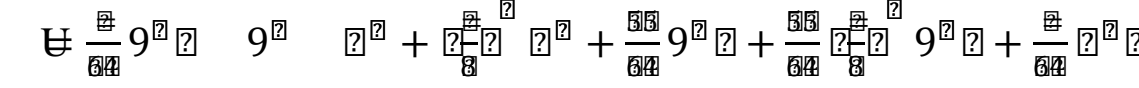

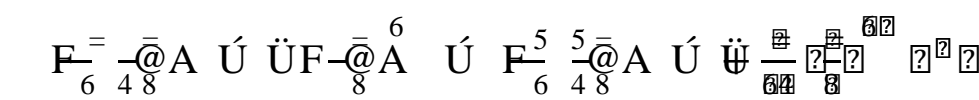

$$
\begin{aligned}
& \mathrm{V}=\frac{55}{64}\left(\begin{array}{ll}
\mathrm{F} 4
\end{array}\right) @ 9 @ \underset{8}{\mathrm{~A}} \mathrm{~F} 1 \mathrm{~A}+4 \mathrm{~A}>0 .
\end{aligned}
$$

For obvious reasons, we resorted to numerical methods to investigate equilibrium conditions DQGUREXVWQHVVZLWKUHVSHFWWR.We summarize our numerical findings for each case.

SymPayAsymRec $(\ell=9)$

For $<=0.9$, we report the numeric solutions for several values of $\ddot{Y}$ in the following table.

Because the equilibrium conditions imply $\ddot{\mathrm{E}}_{5}=\ddot{\mathrm{E}}_{6}=\frac{84}{=}=4.4$, these will not be included. 
Table A.1: Equilibrium probabilities and player 3's payoff in SymPayAsymRec $(\ell=9)$

\begin{tabular}{|c|l|l|l|l|}
\hline$\ddot{\mathrm{Y}}$ & $2 \check{\zeta}_{\overline{5}}$ & $22_{\overline{6}}$ & $2_{7}^{\mathrm{A}}$ & $\ddot{\mathrm{E}}_{7}$ \\
\hline 1.00 & 0.48 & 0.23 & 0.13 & 2.78 \\
\hline 0.90 & 0.63 & 0.28 & 0.21 & 2.25 \\
\hline 0.80 & 0.85 & 0.34 & 0.31 & 1.78 \\
\hline 0.75 & 0.99 & 0.38 & 0.36 & 1.56 \\
\hline
\end{tabular}

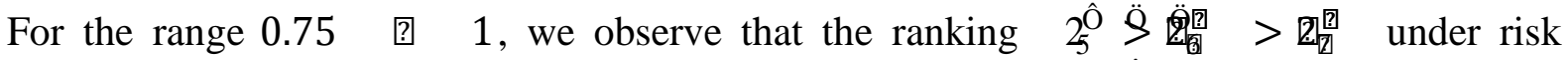
neutrality is preserved under risk aversion. However, probability $2 \stackrel{\leftarrow}{5} \quad$ is less than 1 if and only if $\ddot{Y} \mathrm{R}$ 0.75. Therefore, it is player 1 who is critical in attaining the bound on the risk aversion parameter. Player 3's equilibrium payoff decreases monotonically in the risk aversion parameter.

To summarize, the Nash equilibrium reported in Table 3 is robust for 0.75 Q Ÿ Q 1, its ranking

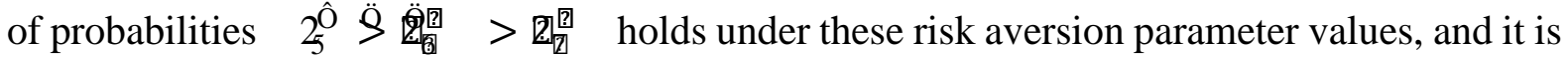
player 1 who becomes pivotal. Player 3's equilibrium payoff decreases monotonically in the risk aversion parameter.

\section{AsymPayAsymRec ( = 15)}

For $<=0.9$, we report the numeric solutions for several values of $\ddot{Y}$ in the following table. Because the equilibrium conditions imply $\ddot{\mathrm{E}}_{5}=\ddot{\mathrm{E}}_{6}=\frac{84}{=}=4.4$, these will not be included.

Table A.2: Equilibrium probabilities and player 3's payoff in AsymPayAsymRec $(\ell=15)$

\begin{tabular}{|c|l|l|l|l|}
\hline$\ddot{\mathrm{Y}}$ & $2 \check{\zeta}$ & $2 \check{\zeta}$ & $2{ }_{7}^{\mathrm{A}}$ & $\ddot{\mathrm{E}}_{7}$ \\
\hline 1.00 & 0.21 & 0.18 & 0.10 & 3.24 \\
\hline 0.90 & 0.28 & 0.22 & 0.18 & 2.70 \\
\hline 0.80 & 0.39 & 0.27 & 0.27 & 2.21 \\
\hline 0.70 & 0.56 & 0.34 & 0.38 & 1.77 \\
\hline 0.60 & 0.81 & 0.42 & 0.51 & 1.37 \\
\hline 0.55 & 0.98 & 0.47 & 0.57 & 1.19 \\
\hline
\end{tabular}

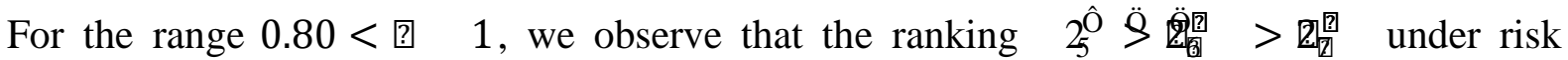
neutrality is preserved under risk aversion, but that this ranking switches to $2 \frac{\dot{\overline{5}}}{2}>2 \stackrel{\overline{\bar{j}}}{>}$ $2 \check{\dot{6}}$ for the range $0.55 \mathrm{Q} \ddot{\mathrm{Y}}<0.80$. The difference between probabilities $2 \dot{\overline{6}}$ and $2 \check{\bar{j}}$ is small and at most 0.10 . Finally, probability $2 \breve{5}$ is less than 1 if and only if $\ddot{\mathrm{Y}} \mathrm{R} 0.55$. Therefore, it is player 1 who is critical in attaining the bound on the risk aversion parameter. Player 3's equilibrium payoff decreases monotonically in the risk aversion parameter.

To summarize, the Nash equilibrium reported in Table 3 is robust for $0.55<\ddot{Y}$ Q 1 , its ranking of probabilities $2 \check{\overline{5}} \quad>2 \check{\overline{6}} \quad>2 \check{\bar{j}}$ holds only for risk aversion parameter values $0.80<\ddot{\mathrm{Y}} \mathrm{Q}$ 
1 , and it is player 1 who becomes pivotal. Player 3's equilibrium payoff decreases monotonically in the risk aversion parameter.

\section{Summary of all four treatments}

To summarize, our closed-form solutions and numerical results indicate that the equilibrium SUREDELOLWLHV GRFKDQJHTXDQWLWDWLYHOIWRFKDQJHVLQWKH\&55\$ULVNFRHIILF However, the investigation of robustness also shows that the hypotheses formulated in the main WH[WDUHTXLWHUREXVWZLWKUHVSHFWWRWKHULVNFRHIILFLHQWSDUDPHWHU. 


\section{Appendix B: Quantal response analysis}

In this analysis we apply the concept of noisy best-response as captured by the quantal response equilibrium to a player's decision whether to propose her best or middle option and whether to accept her middle option. For the case where a player is proposed her best (worst) option we assume that the player does not make any mistakes and follows the intuitively optimal strategy of accepting (rejecting) her best (worst) option. Furthermore, the player will never propose her worst option.

Player E's strategy is therefore described by two probabilities; $2 \div$, the probability of accepting her middle option and $2, \hat{\wedge}$, the probability of proposing her middle option. We will assume that the players have identical CRRA utility functions of the form $Q(T)=T$. We will furthermore use the following notation: $Q$ denotes player Es utility from option $F$; is the probability that player Eis the proposer; < denotes the probability that the game continues WRWKHQH[WSHULRGDIWHUDSURSRVDOKDVEHHQUHMHFWHGDQGLVWKHQRLVHSDUDP

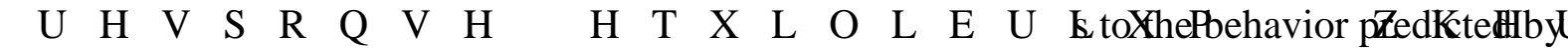
the Nash equilibrium).

The expected utility $\mathrm{E}_{<}$of player Eis then given by:

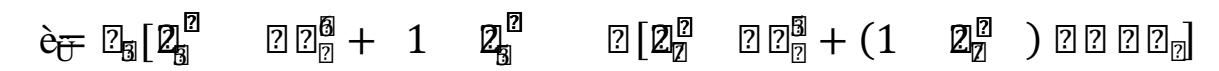

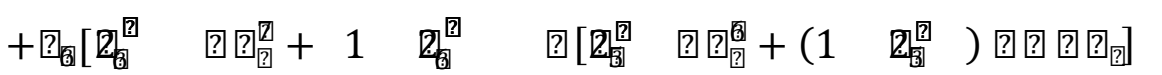

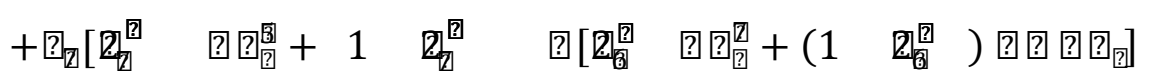

Player Es probability of accepting her middle option is given by: ${ }^{1}$

$$
2 \div=\frac{\exp \left(, € Q^{>5}\right)}{\operatorname{expk}, € Q^{>5} \mathrm{o}+\exp \left(, €<€ \ddot{E}_{\prec}\right)}
$$

where the numerator captures the utility when accepting her middle option and the additional term in the denominator captures the expected utility when rejecting the offer.

Player Es probability of proposing her middle option is given by: ${ }^{2}$

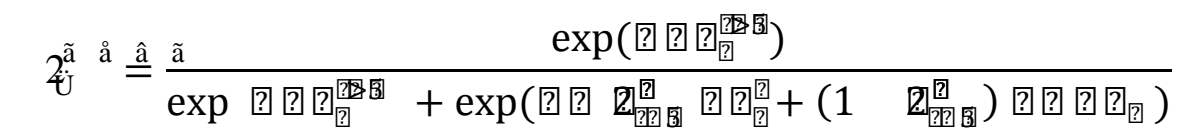

where the numerator captures the utility associated with proposing her (with certainty accepted) middle option and the additional term in the denominator captures the expected utility when proposing her best option.

The three payoff functions and the six equations for the probabilities form a set of equations and the quantal response equilibrium is given by the solution to this fixed point problem. Table

\footnotetext{
${ }^{1}$ For ease of notation we define $\mathrm{Q}^{>5 @ 8}=\mathrm{Q}^{5}$.

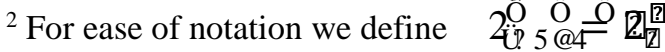


B.1 shows the quantal response equilibrium for the risk-aversion parameter ( $\ddot{Y}=0.47)$ and the noise parameter $(,=2.9)$ that are derived using a maximum likelihood estimation using the data from our experiment.

Table B.1: Quantal response equilibrium

\begin{tabular}{|c|c|c|c|c|}
\hline & SymPaySymRec & SymPayAsymRec & AsymPaySymRec & AsymPayAsymRec \\
\hline \multirow{2}{*}{ Accept } & $86 \%$ & $86 \%$ & $75 \%$ & $76 \%$ \\
M & $86 \%$ & $69 \%$ & $75 \%$ & $59 \%$ \\
& $86 \%$ & $88 \%$ & $85 \%$ & $91 \%$ \\
\hline \multirow{2}{*}{ Propose } & $18 \%$ & $11 \%$ & $12 \%$ & $10 \%$ \\
M & $18 \%$ & $10 \%$ & $3 \%$ & $3 \%$ \\
& $18 \%$ & $24 \%$ & $18 \%$ & $36 \%$ \\
Expected & 4.3 & 3.7 & 4.7 & 4.5 \\
payoff & 4.3 & 5.2 & 6.2 & 7.4 \\
& 4.3 & 3.9 & 3.8 & 3.3 \\
\hline
\end{tabular}

Notes. Cell entries give the probability of accepting (proposing) the middle option and the expected equilibrium payoffs by treatment and player role (the first/second/third entry in each cell corresponds to player 1/2/3) for the TXDQWDOUHVSRQVHHTXLOLEUIDXZLXXK. 7 


\section{Appendix C: Regression analysis for part II}

For the analysis of part II of the experiment we employ logit regressions with the decision to accept or propose one's middle option as the dependent variable and standard errors clustered at the matching group level. To investigate within treatment variations across roles we run regressions with the subject's role in a given round as independent variable. Table C.1 shows the results of this regression by treatment.

Table C.1: Logit regressions by treatment

\begin{tabular}{|c|c|c|c|c|}
\hline & $\begin{array}{c}\text { SymPay } \\
\text { SymRec }\end{array}$ & $\begin{array}{c}\text { SymPay } \\
\text { AsymRec }\end{array}$ & $\begin{array}{c}\text { AsymPay } \\
\text { SymRec }\end{array}$ & $\begin{array}{c}\text { AsymPay } \\
\text { AsymRec }\end{array}$ \\
\hline \multicolumn{5}{|c|}{ Accepting the middle option } \\
\hline Player 2 & -.00 & $-.28^{* * *}$ & $.11^{*}$ & $-.28^{* *}$ \\
\hline Player 3 & .05 & $-.21^{*}$ & $.16^{* *}$ & -.10 \\
\hline \multicolumn{5}{|c|}{ Proposing the middle option } \\
\hline Player 2 & -.02 & $-.13^{* * *}$ & -.01 & $-.06^{* *}$ \\
\hline Player 3 & -.01 & $-.09^{* * *}$ & -.01 & $.05^{*}$ \\
\hline
\end{tabular}

Notes. The table shows the marginal effects of a logit regression with the decision to accept (propose) one's middle option as the dependent variable. 'Player 2' ('Player 3') is equal to one if the player's role is player 2 (player 3$)$. Standard errors are clustered at the matching group level. * $(* * ; * * *)$ denotes that the coefficient is significantly different from zero at the $10 \%$ (5\%; 1\%)-level.

Table C.2: Behavior over time by role and treatment

\begin{tabular}{|l|c|c|c|c|}
\hline & $\begin{array}{c}\text { SymPay } \\
\text { SymRec }\end{array}$ & $\begin{array}{c}\text { SymPay } \\
\text { AsymRec }\end{array}$ & $\begin{array}{c}\text { AsymPay } \\
\text { SymRec }\end{array}$ & $\begin{array}{c}\text { AsymPay } \\
\text { AsymRec }\end{array}$ \\
\hline \multicolumn{5}{|c|}{ Accepting the middle option } \\
\hline Player 1 & -.000 & -.002 & -.030 & -.002 \\
\hline Player 2 & .010 & -.018 & $-.038^{* * *}$ & -.040 \\
\hline Player 3 & .010 & $.014^{* *}$ & -.019 & .011 \\
\hline \multicolumn{5}{|c|}{ Proposing the middle option } \\
\hline Player 1 & -.008 & -.014 & -.011 & -.005 \\
\hline Player 2 & $-.010^{* * *}$ & -.005 & -.005 & -.005 \\
\hline Player 3 & $-.004^{*}$ & -0.05 & -.000 & $.015^{* * *}$ \\
\hline
\end{tabular}

Notes. The table shows the marginal effects of a logit regression with the decision to accept (propose) one's middle option as the dependent variable and the round of the decision as independent variable run separately for each role and treatment. Standard errors are clustered at the matching group level. * (**; ***) denotes that the coefficient is significantly different from zero at the $10 \%$ (5\%; 1\%)-level.

To investigate behavior over time we also ran regressions with the decision round as independent variable for each role and treatment separately. Table C.2 shows the results. As we can see for most roles and treatments there are no significant changes in behavior over time but overall the direction of the change is in line with behavior getting slightly closer to equilibrium over time. The sole exception is the proposal behavior of player 3 in the treatments 
with asymmetric probabilities where the direction of the change is away from the equilibrium choice probabilities.

To investigate the treatments effect on the acceptance and proposing decision table C.3 presents the results from logit regressions with treatment dummies as independent variables run separately by players' role.

Table C.3: Logit regression by role

\begin{tabular}{|c|c|c|c|}
\hline & Player 1 & Player 2 & Player 3 \\
\hline \multicolumn{4}{|c|}{ Accepting the middle option } \\
\hline SymPayAsymRec & .07 & $-.27^{* * *}$ & $-.16^{*}$ \\
\hline AsymPaySymRec & $-.21^{*}$ & -.09 & -.08 \\
\hline AsymPayAsymRec & -.07 & $-.39^{* * *}$ & $-.24^{* * *}$ \\
\hline \multicolumn{4}{|c|}{ Proposing the middle option } \\
\hline SymPayAsymRec & $.14^{* * *}$ & -.01 & .02 \\
\hline AsymPaySymRec & -.03 & -.01 & -.02 \\
\hline AsymPayAsymRec & .02 & -.02 & $.09^{* *}$ \\
\hline
\end{tabular}

Notes. The table shows the marginal effects of a logit regression with the decision to accept (propose) one's middle option as the dependent variable. 'SymPayAsymRec' ('AsymPaySymRec', 'AsymPayAsymRec') is equal to one if the treatment has asymmetric (symmetric, asymmetric) recognition probabilities and the payoffs are symmetric (asymmetric, asymmetric). Standard errors are clustered at the matching group level. * $\left.{ }^{* *}{ }^{* * *}\right)$ denotes that the coefficient is significantly different from zero at the $10 \%$ (5\%; 1\%)-level.

To test the treatment effects regarding the payoffs we ran linear regressions for each role with a player's payoffs as the dependent variable and treatment dummies as independent variables. The results are shown in table C.4.

Table C.4: Effect of treatments on payoffs

\begin{tabular}{|c|c|c|c|}
\hline & Player 1 & Player 2 & Player 3 \\
\hline SymPayAsymRec & $-1.5^{* * *}$ & $2.3^{* * *}$ & $-0.8^{* * *}$ \\
\hline AsymPaySymRec & 0.1 & $1.3^{* *}$ & 0.2 \\
\hline AsymPayAsymRec & $-0.8^{* * *}$ & $4.4^{* * *}$ & $-0.7^{* *}$ \\
\hline
\end{tabular}

Notes. The table shows the coefficients of a linear regression with a player's payoff as the dependent variable. 'SymPayAsymRec' ('AsymPaySymRec', 'AsymPayAsymRec') is equal to one if the treatment has asymmetric (symmetric, asymmetric) recognition probabilities and the payoffs are symmetric (asymmetric, asymmetric). Standard errors are clustered at the matching group level. * $\left.{ }^{* *} ;{ }^{* *}\right)$ denotes that the coefficient is significantly different from zero at the $10 \%$ (5\%; 1\%)-level.

Finally, we investigate whether there are gender effects in data and what effect subjects' elicited risk-aversion has on their decision to propose and accept the middle option. The results shown in Table C.5 indicate that a subject's gender is not an important determinate of behavior since most coefficients are small and insignificant. Furthermore, for the acceptance decision most of the 'risk' coefficients are negative (as we would expect since more risk-averse players have a lower value of the variable 'risk' and are ceteris paribus more likely to accept their middle option) if we aggregate choices over all treatments and roles the coefficient is not 
significant (p-value: 0.41 ). ${ }^{3}$ The same holds true for the proposing behavior where again the majority of the coefficients are negative but overall there is no significant effect of the riskvariable (p-value: 0.54$){ }^{4}$

Table C.5: Effect of risk-aversion and gender

\begin{tabular}{|c|c|c|c|c|c|}
\hline & & $\begin{array}{l}\text { SymPay } \\
\text { SymRec }\end{array}$ & $\begin{array}{l}\text { SymPay } \\
\text { AsymRec }\end{array}$ & $\begin{array}{l}\text { AsymPay } \\
\text { SymRec }\end{array}$ & $\begin{array}{l}\text { AsymPay } \\
\text { AsymRec }\end{array}$ \\
\hline \multicolumn{6}{|c|}{ Accepting the middle option } \\
\hline \multirow{2}{*}{ Player 1} & male & .032 & -.017 & .236 & .015 \\
\hline & risk & -.000 & $-.022^{*}$ & -.027 & .021 \\
\hline \multirow{2}{*}{ Player 2} & male & -.022 & -.091 & $-.170^{*}$ & $-.219^{*}$ \\
\hline & risk & -.006 & -.002 & -.024 & .027 \\
\hline \multirow{2}{*}{ Player 3} & male & -.043 & -.169 & $.157^{*}$ & omitted \\
\hline & risk & $-.021^{* *}$ & .047 & -.074 & -.010 \\
\hline \multicolumn{6}{|c|}{ Proposing the middle option } \\
\hline \multirow{2}{*}{ Player 1} & male & $-.067^{* *}$ & -.036 & .036 & -.012 \\
\hline & risk & .002 & .003 & -.016 & .006 \\
\hline \multirow{2}{*}{ Player 2} & male & -.056 & .018 & -.049 & -.020 \\
\hline & risk & -.007 & -.015 & .003 & -.008 \\
\hline \multirow{2}{*}{ Player 3} & male & -.017 & .056 & .000 & -.024 \\
\hline & risk & -.003 & -.017 & -.014 & .004 \\
\hline
\end{tabular}

Notes. The table shows the marginal effects of a logistic regression with the decision to accept (propose) one's middle option as the dependent variable. 'Male' is equal to one if the subject is male and 'risk' is equal to the choice made in the risk-elicitation task in part III of the experiment (possible values are 1-7 where higher number indicate less risk-averse preferences). Standard errors are clustered at the matching group level. * (**; ***) denotes that the coefficient is significantly different from zero at the $10 \%$ (5\%; $1 \%)$-level.

\footnotetext{
${ }^{3}$ If we aggregate choices only over treatments but run regression for separate roles we find that the effect of 'risk' is only significantly negative for player 3 with p-values of $0.96(0.77 ; 0.05)$ for player $1(2 ; 3)$. When aggregating over players and running separate regressions by treatments the only significant effect is a positive coefficient in treatment AsymPayAsymRec (p-value $<0.01$ ) while in the other three treatments the coefficients are negative and insignificant (SymPaySymRec: 0.25; SymPayAsymRec: 0.87; AsymPaySymRec: 0.43).

${ }^{4}$ If we aggregate choices only over treatment and have separate regressions for different roles all coefficients are insignificant (player 1: 0.63; player 2: 0.11; player 3: 0.30). The same holds true for aggregating by treatment (SymPaySymRec: 0.72; SymPayAsymRec: 0.50; AsymPaySymRec: 0.27; AsymPayAsymRec: 0.96)
} 


\section{Appendix D: Best responses against observed fractions}

In this appendix we investigate to what degree subjects' behavior can be explained by them best-responding to the other players' behavior. To study this question we use observed behavior in each independent matching group to compute the expected continuation value of rejecting one's middle option and proposing one's best option. To take account of possibly risk-averse agents we once compute these continuation values assuming risk-neutrality and once assuming a utility function $7(\mathrm{~T})=æ \overline{\mathrm{T}}$, which is quite close to risk-aversion estimated using the data. First we consider the decision whether to accept one's middle option (as shown in Figure D.1) and then we investigate proposing behavior (as shown in Figure D.2).

\section{Acceptance decision}

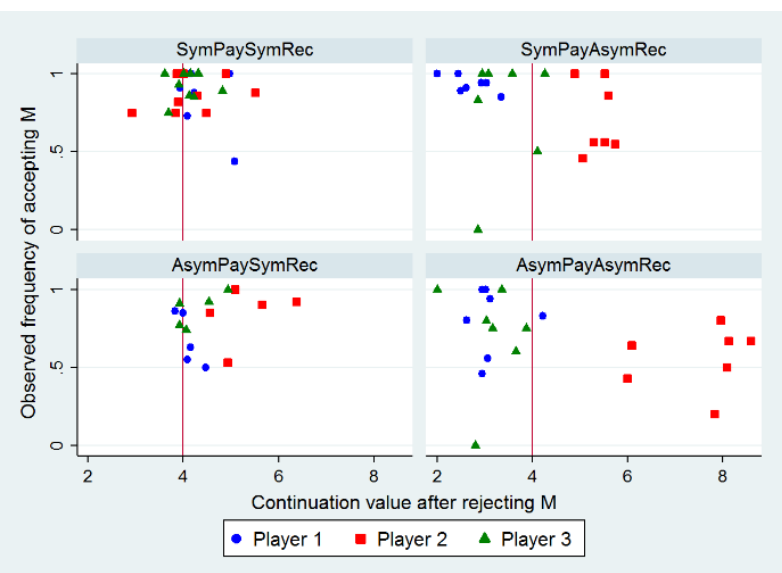

(a) Risk-neutral case: $7(\mathrm{~T})=\mathrm{T}$

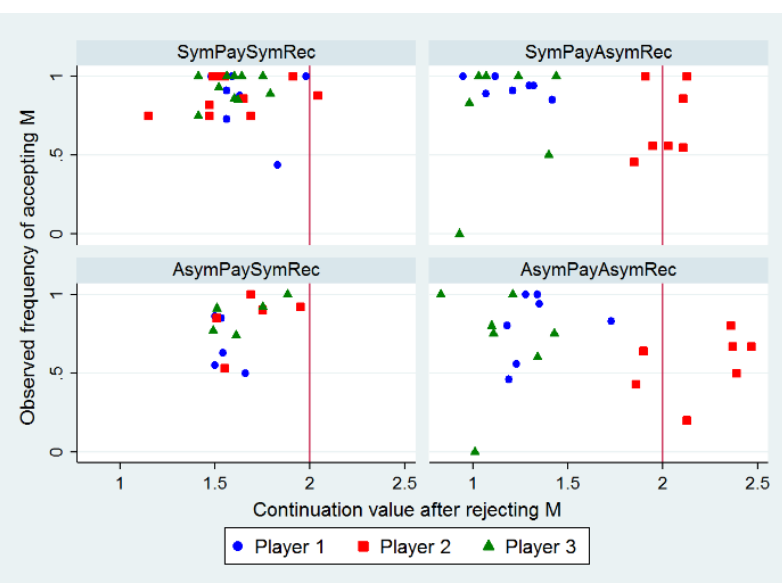

(b) Risk-averse case: $7(\mathrm{~T})=æ \overline{\mathrm{T}}$

Figure D.1: Observed acceptance rates and continuation values

Notes. The figure show for each matching group and player type the observed acceptance rates of their middle option and their continuation values after rejecting their middle option. Panel (a) shows the continuation values for a risk-neutral agent and panel (b) for a risk-averse agent. The vertical line represents the value of accepting. Theoretically, the best response is 1 (0) for continuation values to the left (right) of this line, and randomization on this line.

In treatment SymPaySymRec we observe that risk-neutral subjects are accepting their middle option too often but most of these mistakes are at moderate costs. When we then consider riskaverse agents we find that the high probability of accepting one's middle option is in line with best-responding to observed behavior.

When we go from the symmetric situation to treatment SymPayAsymRec, we see that Player 2 is clearly distinct from the other two types. While both Player 1 and Player 3 are bestresponding to observed behavior ${ }^{5}$ (both when being risk-neutral or risk-averse), risk-neutral player 2's are accepting their middle option too often even though this comes at a substantial cost to them. Again, assuming risk-aversion brings the data closer to being a best-response.

\footnotetext{
${ }^{5}$ The data point showing a Player 3 having a zero acceptance rate is due to the fact that in one matching group this player type only got a single proposal which was rejected.
} 
Overall, this analysis reveals that while Player 1's higher than the equilibrium acceptance rate can be explained by being a best-response to other players' behavior, this explanation has less bite looking at Player 2's behavior.

In treatment AsymPaySymRec we have a similar picture as in SymPayAsymRec. If players are risk-neutral we observe Player 2 accepting her middle option too frequently while the other two types are playing consistent with best-responding to observed behavior. If we assume riskaverse agents we can explain Player 2's high acceptance rate but now Player 1 is rejecting her middle option too often. Overall, the deviations from Nash equilibrium by players 1 and 2 can only be partially explained by being a best-response to observed behavior.

In AsymPayAsymRec we observe the sharpest difference between Player 2 and the other two types. Player 2 is accepting her middle option too frequently and this comes at very big costs. Furthermore, this deviation from best-responding cannot be explained by risk-aversion and at the same time with risk-averse players the other two types are rejecting their middle option too often. ${ }^{6}$

From the analysis of the acceptance behavior three conclusions emerge. First, in the presence of asymmetries Player 2 is accepting her middle option substantially more often than she should given the other players' behavior and therefore the observed deviations from the Nash equilibrium cannot be explained by being a best-response. Second, while assuming riskaversion brings Player 2's behavior closer to being a best-response to observed behavior, in the AsymPay treatments player 1 is now rejecting her middle option too frequently. Third, with asymmetric recognition probabilities player 2's behavior is noticeably different from the other two types since accepting her middle option comes at much larger costs than for the other two types.

\section{Proposing decision}

In treatment SymPaySymRec we observe that players propose their middle option more often than would be a best-response and allowing for risk-aversion does not change this result. A similar picture emerges in SymPayAsymRec where Player 3 is closest to best-responding to observed behavior and makes the least costly mistakes. We also see that the observed high rate of proposing her middle option by player 1 is not a best-response to observed behavior whether agents are risk-averse or not.

While in the treatments with symmetric payoffs there is little relation between the cost of an error and its frequency, in AsymPaySymRec we see such a negative relationship. Overall, all types have a low frequency of proposing their middle option which is a best-response to observed behavior. The negative relationship between costs and frequency of proposing one's middle option is even stronger in AsymPayAsymRec and the comparative statics across players are organized well by these costs. In general proposing behavior in this treatment is quite close to a best-response.

\footnotetext{
${ }^{6}$ The data point showing a Player 3 having a zero acceptance rate is due to the fact that in one matching group this player type only got two proposals which were both rejected.
} 
Combining what we found in the analysis of the proposing behavior we can draw four conclusions. First, players overall propose their middle option too often given observed behavior and given that in almost all matching groups it would have been a best-response to always propose one's favorite option. Second, even allowing for risk-aversion does not change the previous conclusion. Third, with symmetric payoffs the continuation value after proposing one's best option has little influence on the frequency of proposing one's middle option while with asymmetric payoffs we see a negative relationship. Fourth, with asymmetric recognition probabilities Player 1's behavior is farthest away from being a best-response to the observed behavior.

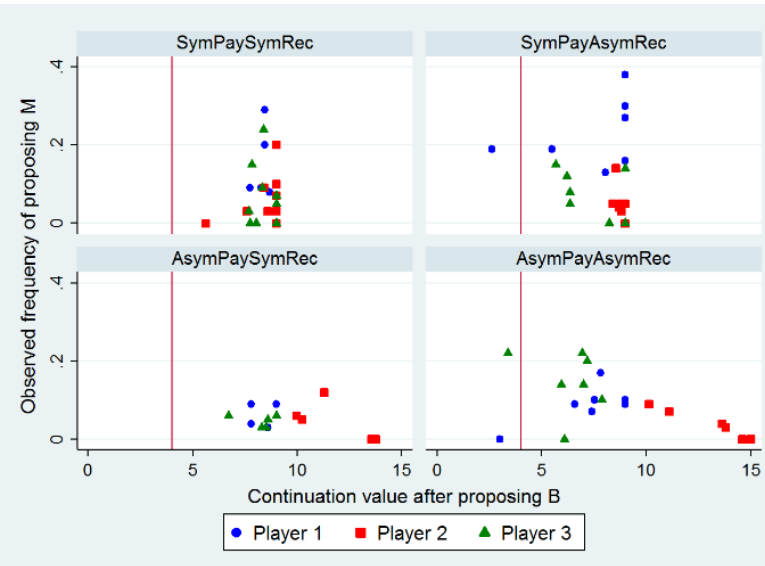

(a) Risk-neutral case: $7(\mathrm{~T})=\mathrm{T}$

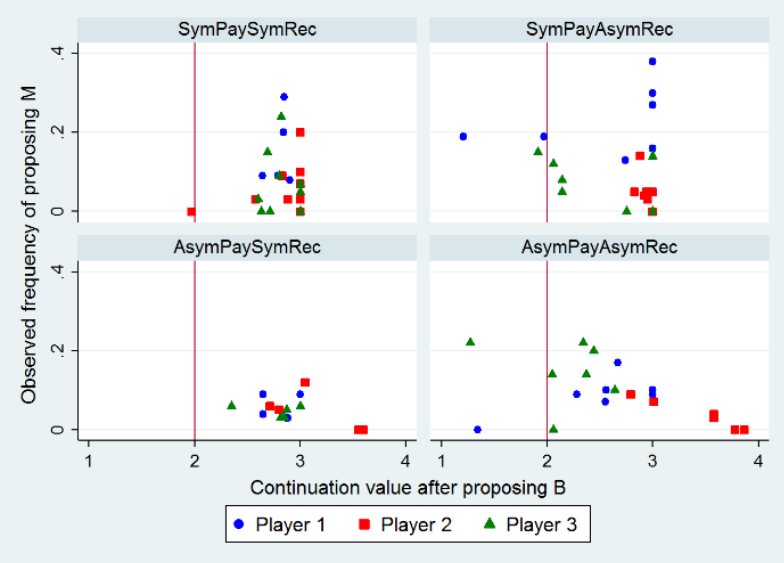

(b) Risk-averse case: $7(\mathrm{~T})=\mathfrak{} \overline{\mathrm{T}}$

Figure D.2: Observed proposal rates and continuation values

Notes. The figure show for each matching group and player type the observed proposal rates of their middle option and their continuation values after proposing their favorite option. Panel (a) shows the continuation values for a risk-neutral agent and panel (b) for a risk-averse agent. The vertical line represents the value of proposing the middle alternative, which is immediately accepted. Theoretically, the best response is 0 (1) for continuation values to the right (left) of this line, and randomization on this line. 


\section{Appendix E: Behavior in part I}

In this appendix, we present a short analysis of behavior in part I of the experiment. Overall, subjects accept and propose their middle option more often than predicted, which leads to faster agreement than predicted (see Figure E.1). As a result we have only few observations of proposing behavior and even fewer acceptance decisions (for instance in treatment SymPaySymRec not a single player 1 was proposed her middle option ${ }^{7}$ ). We therefore do not investigate the differences in behavior using a detailed statistical analysis but instead focus on three stylized facts that we compare to what we find in part II of the experiment.

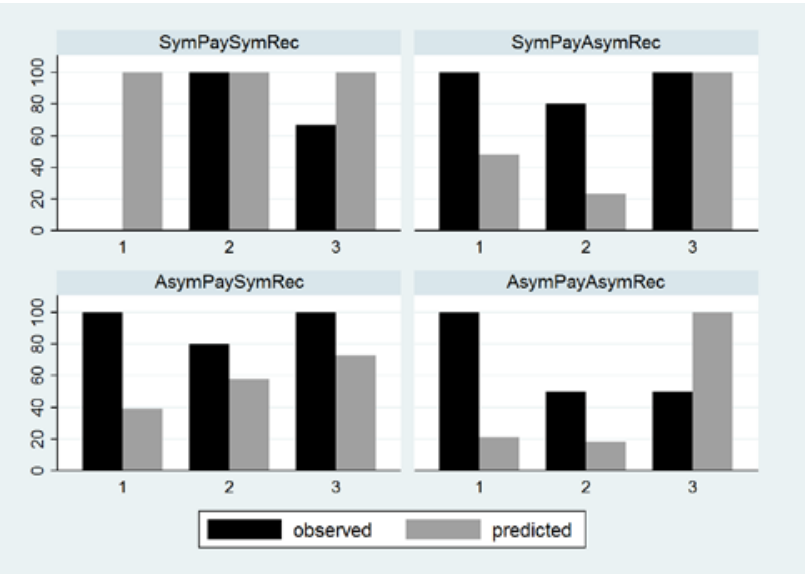

(a) fraction accepting middle option

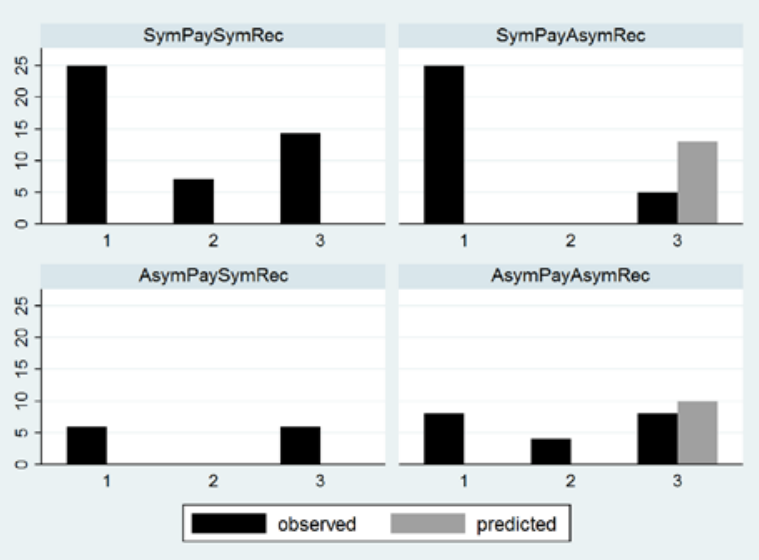

(b) fraction proposing middle option

Figure E.1: Accepting and Proposing one’s Middle Option in Part I

Notes. The figure shows the average fraction of players accepting and proposing their middle option observed in part I of the experiment, split by role and treatment and compares them to the Nash equilibrium predictions.

First, the already mentioned higher overall acceptance rates are especially pronounced for player 1 . For instance when she is the 'weak' player who has a low recognition probability, she always accepts her middle option while in the predicted equilibrium she should frequently reject her middle option. As discussed in section 3.1, a similar pattern is also observed in part II of the experiment. Second, when the game is completely symmetric (SymPaySymRec) players frequently propose their middle option while they are predicted to always propose their best option. This may be because with the high payoffs in part I, breakdown would be socially very costly and the payoff of one's second favorite option is still substantial. This, in addition to learning, would also explain why in part II of the experiment we observe behavior that is closer to equilibrium (there, players almost always proposed their best option). Third, in treatment SymPayAsymRec it is not (the predicted) player 3 that is mostly likely to propose her middle option. Instead, and similar to part II of the experiment, player 1 very frequently proposes her middle option.

\footnotetext{
${ }^{7}$ Recall that alternative II is the middle option for player 1 . It is the best option for player 2, who hardly ever proposes it.
} 


\section{Appendix F: Instructions and screenshots of the experiment}

In this appendix, we provide the instructions that the subjects read on their monitors. We also give the summary of the instructions that was handed out to subjects after they had read these on-screen instructions. Finally, we provide screenshots of the user interface of the experiment.

\section{F.1 Instructions ${ }^{8}$}

Welcome to this experiment on decision-making. Please carefully read the following instructions. If you have any questions, please raise your hand, and we will come to your table to answer your question in private.

In this experiment you will earn points. At the end of the experiment, your earnings in points will be exchanged for money at rate 10 eurocent for each point. This means that for each 10 points you earn, you will receive 1 euro. Additionally, you will receive a show-up fee of 7 euros. Your earnings will be privately paid to you in cash at the end of the experiment.

This experiment consists of 3 parts. You will first receive the instructions for the first part. The instructions for the second part you will only receive once the first part is done. The instructions for the third part you will receive after the second part is done.

\section{Instructions for part I}

In the first part of the experiment you will be randomly matched with two other persons in the lab with whom you will never interact in parts II and III of this experiment. Your group of three consists of a player 1, a player 2 and a player 3 . These roles are randomly determined in the beginning of the first part of the experiment and the roles stay the same throughout the first part of the experiment.

The task that the group has to perform is to select one out of three alternatives that then determines the payoffs in this round. In the table below you see the payoffs assigned to each type of player by the different alternatives. Remember that each point is worth 10 cents.

\begin{tabular}{|c|c|c|}
\hline Alternative 1 & Alternative 2 & Alternative 3 \\
\hline Player1: 90 points & Player1: 40 points & Player1: 0 points \\
\hline Player 2: 0 points & Player 2: 150 points & Player 2: 40 points \\
\hline Player 3: 40 points & Player 3: 0 points & Player 3: 90 points \\
\hline
\end{tabular}

The process of choosing an alternative is organized by periods. In each period all group members submit a proposal (being one of the three alternatives) they want the other group members to vote on. After every group member has submitted a proposal one of the proposals is randomly chosen to be voted on. The probabilities for the different players are presented in the table below.

\footnotetext{
${ }^{8}$ We provide here the instructions used for the treatment AsymPaySymRec. The instructions for other treatments are analogous and available upon request.
} 


\begin{tabular}{|c|c|c|}
\hline Player 1 & Player 2 & Player 3 \\
\hline $33.3 \%$ & $33.3 \%$ & $33.3 \%$ \\
\hline
\end{tabular}

As you can see the proposal by player 1 has a lower chance of being put up for a vote than the a proposal by players 2 or 3 .

The proposal of the selected player (the "proposer") is then communicated to the other two group members which then can vote to accept or reject the proposal. The voting procedure works as follows: First the player who earns a higher payoff from the proposal gets to cast his vote. Given that the proposer supports his own proposal the proposal is accepted if the first voter accepts the proposal. In this case the first part of the experiments ends and the payoffs for this part are computed according to the chosen alternative. After this the experiment moves to the second part of the experiment.

If the first voter rejects the offer the remaining group member (who is not the proposer) gets to cast his vote. If he votes yes the proposal is accepted, the payoffs for this part are computed according to the chosen alternative and the experiments moves to the second part.

Should also the second group member reject the proposal two things can happen: With probability $90 \%$ the game continues to the next period and again proposals have to be submitted. With a $10 \%$ chance the game ends after a proposal was rejected and payoffs for the first part are zero for all group members. Furthermore the experiment moves to part II.

Before the first part of the experiment starts, please answer the questions on this page.

1. Assume that in the current period you are in the same group as subjects 3 and 10 . Will you be in the same group as subject 10 in the next period? $<$ Yes/No $>$

2. Assume that in the current period you are a player 1. Will you be the same player in the next period? $<$ Yes/No $>$

3. $\quad$ Can there be two player 2 in the same group? $<$ Yes/No $>$

4. What is the payoff (in points) to player 1 if alternative 1 is chosen?

5. What is the payoff (in points) to player 2 if alternative 2 is chosen?

6. In each period you select an alternative that you want to propose. Is it $100 \%$ certain that that your alternative will be selected and voted on by the other players? $<$ Yes/No $>$

7. Suppose that player 1 proposes alternative 2; who will be the first player to vote on the proposal? < Player 1/Player 2/Player 3>

8. Suppose that player 3 proposes alternative 3; who will be the first player to vote on the proposal? < Player 1/Player 2/Player 3> 
9. Suppose that player 1 proposes alternative 1 ; will player 2 be casting a vote in this case? $<$ Yes/No/Depends on player 3's voting decision>

10. What happens if the proposal is rejected? < The experiment continues to part II and you get a payoff of zero/The game continues to the next period/Both can happen>

11. Will you interact with the two players you are grouped with in part I at any point during part II and part III? < Yes/No>

\section{Instructions for part II}

The second part of the experiment consists of 10 rounds. In each round you will play a similar game to the one in part I.

In each round you will be randomly matched with two other persons in the lab (that can not be the same persons you interacted with in part I). Again, a group of three always consists of a player 1, a player 2 and a player 3. These roles are randomly determined in every round. This means, for instance, that you can be player 1 in one round and player 2 in another round.

As in part I the task that the group has to perform is to select one out of three alternatives that then determines the payoffs in this round. In the table below you see the payoffs assigned to each type of player by the different alternatives. Please note that the payoffs are different from part I.

\begin{tabular}{|c|c|c|}
\hline Alternative 1 & Alternative 2 & Alternative 3 \\
\hline Player1: 9 points & Player1: 4 points & Player1: 0 points \\
\hline Player 2: 0 points & Player $2: 15$ points & Player $2: 4$ points \\
\hline Player 3: 4 points & Player 3: 0 points & Player $3: 9$ points \\
\hline
\end{tabular}

The process of choosing an alternative is organized as in part I. As a reminder: This means that in each period all group members submit a proposal (being one of the three alternatives) they want the other group members to vote on. After every group member has submitted a proposal one of the proposals is randomly chosen to be voted on. The probabilities for the different players are presented in the table below and are the same as in part I.

\begin{tabular}{|c|c|c|}
\hline Player 1 & Player 2 & Player 3 \\
\hline $33.3 \%$ & $33.3 \%$ & $33.3 \%$ \\
\hline
\end{tabular}

The game proceeds than in the same fashion as in part I: The proposal of the selected player is communicated to the other two group members which then can vote to accept or reject the proposal. First the player who earns a higher payoff from the proposal gets to cast his vote. If this first voter accepts the proposal the proposal is accepted. In this case the round ends and the payoffs for this round are computed according to the chosen alternative. After this the experiment moves to the next round.

If the first voter rejects the offer the remaining group member (who is not the proposer) gets to cast his vote. If he votes yes the proposal is accepted and the experiments moves to the next round. 
Should also the second group member reject the proposal two things can happen: As in part I with probability $90 \%$ the round continues to the next period and again proposals have to be submitted. With a $10 \%$ chance the rounds ends after a proposal was rejected and payoffs for this round are zero for all group members. Furthermore the experiment moves to the next round.

After all 10 rounds have passed the payoffs from all rounds are added it up and exchanged at a rate of 10 cent per point.

Before the experiment starts, please answer the questions on this page.

1. Assume that in the current round you are in the same group as subjects 3 and 10 . Will you be in the same group as subject 10 in the next round? <Yes/No/Impossible to know since there is random rematching $>$

2. Assume that in the current period you are in the same group as subjects 3 and 10 . Will you be in the same group as subject 10 in the next period? <Yes/No/Impossible to know since there is random rematching>

3. Assume that in the current round you are a player 1. Will you be the same player in the next round? < Yes/No/Impossible to know since there is random rematching >

4. Assume that in the current period you are a player 1. Will you be the same player in the next period? $<$ Yes/No/Impossible to know since there is random rematching $>$

5. What happens if the proposal is accepted? < The experiment continues to a new round/The experiment stays in the same round but goes to the next period>

6. What happens if the proposal is rejected? < The experiment continues to a new round and you get a payoff of zero/The experiment stays in the same round and goes to the next period/Both can happen>

\section{Instructions for part III}

The third part of the experiment only consists of the choice described below. Again each point is worth ten cent.

In the table below, we present six different options. Please select one of the options.

Your earnings will depend on the outcome of a fair coin toss. Every option shows the amount in points you earn in case a head shows up or a tail shows up.

When determining your total earnings for this experiment, the computer will "toss a coin" and add an amount according to the outcome of the toss and the choice you made to your earnings of parts 1 and 2. The outcome of the coin toss will be determined after you submitted your choice and will be shown to you on the next page. 


\begin{tabular}{|c|c|c|}
\hline & $\begin{array}{l}\text { Your earnings when coin } \\
\text { indicates heads }\end{array}$ & $\begin{array}{l}\text { Your earnings when coin } \\
\text { indicates tails }\end{array}$ \\
\hline Option 1 & 25 points & 25 points \\
\hline Option 2 & 33 points & 21 points \\
\hline Option 3 & 41 points & 17 points \\
\hline Option 4 & 49 points & 13 points \\
\hline Option 5 & 57 points & 9 points \\
\hline Option 6 & 62 points & 5 points \\
\hline Option 7 & 65 points & 0 points \\
\hline
\end{tabular}




\section{F.2 Printed summary of instructions}

\section{Summary instructions: Part I}

$x$ The experiment consists of three parts; these are the instructions for the first part

$\mathrm{X}$ You will be in a group of three players. A group always consists of a player 1, a player 2 and a player 3.

$\mathrm{x}$ For the whole first part you will be player 1 or player 2 or player 3 .

$\mathrm{x}$ Your task is to decide which of three alternatives (see below) to implement

\begin{tabular}{|l|c|c|c|}
\hline & Alternative 1 & Alternative 2 & Alternative 3 \\
\hline Payoff player 1 & 90 & 40 & 0 \\
\hline Payoff player 2 & 0 & 90 & 40 \\
\hline Payoff player 3 & 40 & 0 & 90 \\
\hline
\end{tabular}

$x$ The process of choosing an alternative is organized by periods. In each period every player will propose an alternative to the other two players. The proposal of only one player will be randomly chosen to be voted upon and then be shown to the other two players.

$\mathrm{X}$ The probability that a given player's proposal is chosen is given below

\begin{tabular}{|l|c|c|c|}
\hline & Player 1 & Player 2 & Player 3 \\
\hline probability & $33.3 \%$ & $33.3 \%$ & $33.3 \%$ \\
\hline
\end{tabular}

$\mathrm{x}$ The voting procedure works as follows:

1. Out of the two players who are not the proposer, the player who has a higher payoff from the proposal gets to cast his vote first. If he accepts this proposal is implemented.

2. If the proposal gets rejected by the first voter the other player who is not the proposer gets to cast his ballot. If he votes "Yes" the proposal is accepted. If he also votes "No" the proposal is rejected.

$x$ If the proposal is accepted, this proposal is implemented and everyone gets the payoffs associated with this alternative. The first part of the experiment ends then.

$\mathrm{X}$ If the proposal is rejected, two things can happen:

1. In 1 out of 10 cases: the first part of the experiment ends and everyone receives a payoff of zero for this part.

2. In 9 out of 10 cases: the game continues to the next period where again proposals are made and voted upon. 
At the end of the experiment each point is worth ten cents and together with a show-up fee of $7 €$ you will receive these earnings in private at the end of the experiment together with your earnings of parts two and three of the experiment.

\section{Summary instructions: part II}

$x$ The experiment consists of three parts; these are the instructions for the second part

$\mathrm{x}$ This part consists of 10 rounds

$x$ In each round you will be in a group of three players. A group always consists of a player 1, a player 2 and a player 3 .

$x \quad$ In each round you will be player 1 or player 2 or player 3 .

$x$ After each round you get randomly rematched with two other persons in the lab and be randomly assigned player 1 or player 2 or player 3.

$x$ Your task in each round is to decide which of three alternatives (see below) to implement

\begin{tabular}{|l|c|c|c|}
\hline & Alternative 1 & Alternative 2 & Alternative 3 \\
\hline Payoff player 1 & 9 & 4 & 0 \\
\hline Payoff player 2 & 0 & 9 & 4 \\
\hline Payoff player 3 & 4 & 0 & 9 \\
\hline
\end{tabular}

X The process of choosing an alternative is organized by periods. In each period every player will propose an alternative to the other two players. The proposal of only one player will be randomly chosen to be voted upon and then be shown to the other two players.

$\mathrm{X}$ The probability that a given player's proposal is chosen is given below

\begin{tabular}{|l|c|c|c|}
\hline & Player 1 & Player 2 & Player 3 \\
\hline probability & $33.3 \%$ & $33.3 \%$ & $33.3 \%$ \\
\hline
\end{tabular}

$\mathrm{x}$ The voting procedure works as follows:

3. Out of the two players who are not the proposer, the player who has a higher payoff from the proposal gets to cast his vote first. If he accepts this proposal is implemented.

4. If the proposal gets rejected by the first voter the other player who is not the proposer gets to cast his ballot. If he votes "Yes" the proposal is accepted. If he also votes "No" the proposal is rejected.

$x$ If the proposal is accepted, this proposal is implemented and everyone gets the payoffs associated with this alternative. 
$\mathrm{x}$ If the proposal is rejected, two things can happen:

3. In 1 out of 10 cases: the round ends and everyone receives a payoff of zero for this round.

4. In 9 out of 10 cases: the round continues to the next period where again proposals are made and voted upon.

$x$ At the end of the experiment each point is worth ten cents and together with a show-up fee of $7 €$ you will receive these earnings in private at the end of the experiment together with your earnings of parts one and three of the experiment. 


\section{F.3 Screenshots of the interface}

This is period 1 in round 1

You are player 1

Which alternative do you want to propose?

\begin{tabular}{|c||c|c|}
\hline & & \\
\hline $\begin{array}{c}\text { Player } 1: 90 \text { points } \\
\text { Player 2: } 0 \text { points }\end{array}$ & Player $1: 40$ points & Player $1: 0$ points \\
Player 3: 40 points & Player 2: 90 points & Player 2: 40 points \\
& Player 3: 90 points \\
Alternative 1 & CAlternative 2 & Alternative 3 \\
\hline
\end{tabular}

Submit

Notes. The screen subjects saw when making a decision for which option to propose.

This is period 2 in round 3

You are player 1

Which alternative do you want to propose?

\begin{tabular}{|c|c|c|}
\hline $\begin{array}{l}\text { Player } 1: 90 \text { points } \\
\text { Player 2: } 0 \text { points } \\
\text { Player 3: } 40 \text { points }\end{array}$ & $\begin{array}{l}\text { Player 1: } 40 \text { points } \\
\text { Player 2: } 90 \text { points } \\
\text { Player 3: } 0 \text { points }\end{array}$ & $\begin{array}{l}\text { Player 1: } 0 \text { points } \\
\text { Player 2: } 40 \text { points } \\
\text { Player } 3: 90 \text { points }\end{array}$ \\
\hline Alternative 1 & Alternative 2 & Alternative 3 \\
\hline
\end{tabular}

Submit

\begin{tabular}{|c||c|c||c|c||c|}
\hline \multicolumn{1}{|c|}{ Round } & Period & Role & Proposal & Your decision & Result \\
\hline 1 & 1 & 1 & 0 & Proposer & Alternative 2 chooscn \\
\hline
\end{tabular}

Notes. The screen subjects saw when making a decision for which option to propose; the table at the bottom of the screen shows an example of the history box.

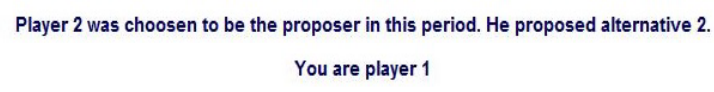

Do you want to accept this proposal??

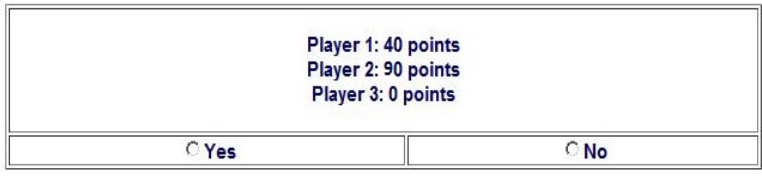

Submit

Notes. The screen subjects saw when deciding whether to accept a proposal. 


\title{
Proposal in period 1
}

\author{
Player 2's proposal was accepted by both players. Therefore alternative 2 will be implemented. \\ Your resulting payoff in this round is therefore 0 points. \\ Your accumulated payoff after this round is 0 points. \\ Click here to go to the next round.
}

Notes. The screen subjects saw after a proposal was accepted.

\section{Proposal in period 1}

The proposal was rejected by both players.

Click here to go to the next period.

Notes. The screen subjects saw after a proposal was rejected.

The bargaining broke down. Therefore you do not earn any points from this rounds.

Your accumulated payoff after this round is 0 points.

Click here to go to the next round.

Notes. The screen subjects saw when bargaining broke down. 